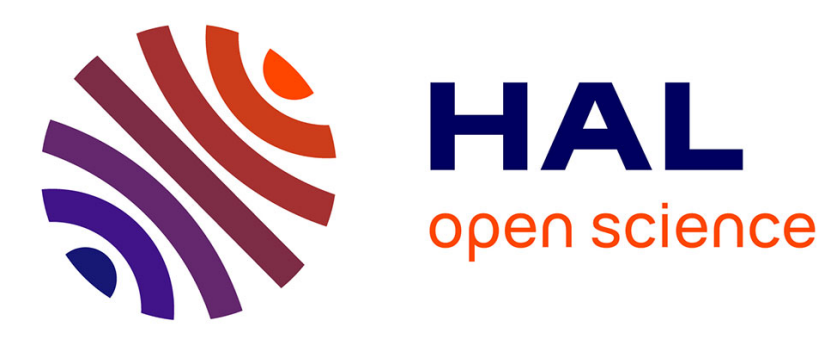

\title{
Mollification in strongly Lipschitz domains with application to continuous and discrete de rham complexes
}

\author{
Alexandre Ern, Jean-Luc Guermond
}

\section{- To cite this version:}

Alexandre Ern, Jean-Luc Guermond. Mollification in strongly Lipschitz domains with application to continuous and discrete de rham complexes. Computational Methods in Applied Mathematics, 2016, 16 (1), pp.51-75. 10.1515/cmam-2015-0034 . hal-01192941v2

\section{HAL Id: hal-01192941 \\ https://hal.inria.fr/hal-01192941v2}

Submitted on 6 Apr 2018

HAL is a multi-disciplinary open access archive for the deposit and dissemination of scientific research documents, whether they are published or not. The documents may come from teaching and research institutions in France or abroad, or from public or private research centers.
L'archive ouverte pluridisciplinaire HAL, est destinée au dépôt et à la diffusion de documents scientifiques de niveau recherche, publiés ou non, émanant des établissements d'enseignement et de recherche français ou étrangers, des laboratoires publics ou privés. 


\title{
MOLLIFICATION IN STRONGLY LIPSCHITZ DOMAINS WITH APPLICATION TO CONTINUOUS AND DISCRETE DE RHAM COMPLEXES ${ }^{* \S}$
}

\author{
ALEXANDRE ERN ${ }^{\dagger}$ AND JEAN-LUC GUERMOND $\ddagger$
}

\begin{abstract}
We construct mollification operators in strongly Lipschitz domains that do not invoke non-trivial extensions, are $L^{p}$ stable for any real number $p \in[1, \infty]$, and commute with the differential operators $\nabla, \nabla \times$, and $\nabla \cdot$. We also construct mollification operators satisfying boundary conditions and use them to characterize the kernel of traces related to the tangential and normal trace of vector fields. We use the mollification operators to build projection operators onto general $H^{1}-, \boldsymbol{H}$ (curl)and $\boldsymbol{H}$ (div)-conforming finite element spaces, with and without homogeneous boundary conditions. These operators commute with the differential operators $\nabla, \nabla \times$, and $\nabla \cdot$, are $L^{p}$-stable, and have optimal approximation properties on smooth functions.
\end{abstract}

Key words. Finite element approximation, mollification, De Rham diagram

AMS subject classifications. 65D05, 65N30, 41A65

1. Introduction. Smoothing by mollification is an important tool for the analysis and the approximation of partial differential equations. This tool has been introduced by Leray [24, p. 206], Soboleff [31, p. 487], and Friedrichs [18, p. 136-139]. The mollifying operation commutes with differential operators and converges optimally when the function to be smoothed is defined over the entire space $\mathbb{R}^{d}$. These properties may not be easy to achieve if the function in question is defined only in a non-smooth domain $D$, say only Lipschitz, since mollification by convolution as originally introduced in the above references requires to extend the function outside $D$, which is a non-trivial task in general unless the boundary and the function are reasonably smooth. The boundary difficulty has been overcome by Blouza and Le Dret [5] and Girault and Scott [19] by redefining mollification using a convolutiontranslation technique so that mollification does not require information outside of $D$. The question is, however, more subtle when dealing with vectors fields where normal or tangent boundary conditions are involved, and, to our knowledge, has not yet been fully addressed in the literature.

The first objective of this paper is to revisit the theory of mollification for scalarand vector-valued fields in strongly Lipschitz domains with the following goals in mind: the mollification operators must be compatible with the De Rham complex (i.e., they must commute with the standard differential operators $\nabla, \nabla \times$, and $\nabla \cdot$ ), be $L^{p}$-stable for any real number $p \in[1, \infty]$, and have strong convergence properties in the entire domain. Using a partition of unity subordinated to a covering of the boundary as done in Blouza and Le Dret [5] and Girault and Scott [19] is (seemingly) incompatible with the first constraint. The route that we propose instead is based on a shrinking technique of the domain using transversal vector fields. We also devise

*This material is based upon work supported in part by the National Science Foundation grants DMS-1015984 and DMS-1217262, by the Air Force Office of Scientific Research, USAF, under grant/contract number FA99550-12-0358. Draft version, April 5, 2018

†Université Paris-Est, CERMICS (ENPC), 77455 Marne-la-Vallée cedex 2, France.

$\ddagger$ Department of Mathematics, Texas A\&M University 3368 TAMU, College Station, TX 77843, USA

$\S$ Published: A. Ern, J.-L. Guermond, Mollification in strongly Lipschitz domains with application to continuous and discrete De Rham complex, Computational Methods in Applied Mathematics, Volume 16, Issue 1, (2016) 51-75. 
a second sequence of mollification operators based on the extension by zero; the corresponding operators have the property that the mollified functions are in the kernel of the canonical trace operators in $H^{1}, \boldsymbol{H}$ (curl), and $\boldsymbol{H}$ (div) (i.e., zero trace, zero tangential trace, and zero normal trace, respectively). The main results of the first part of the paper are Theorems 3.3 and 4.4, and their respective corollaries. As an application of independent interest, we give in Theorem 4.7 a clear characterization of the kernel of the traces associated with the divergence and the curl operators.

The second objective of this work is to use the mollification operators introduced in the first part of the paper to construct quasi-interpolation operators onto general finite element subspaces of $H^{1}, \boldsymbol{H}(\operatorname{curl})$, and $\boldsymbol{H}$ (div), with and without homogeneous boundary conditions. We want these operators to satisfy three key properties: (i) $L^{p}$-stability for any $p \in[1, \infty]$, (ii) commutation with differential operators, (iii) preservation of functions in the finite element spaces. Operators with such properties are important tools in finite element exterior calculus, see Arnold et al. [2, §5.4], $[3, \S 5.3]$, where they are termed bounded cochain projections. In particular, the above properties imply that the quasi-interpolation error is bounded by the best approximation error.

The bases for constructing stable, commuting, and quasi-interpolation projections have been laid out in Schöberl [28, 30] and Christiansen [10], where stability and commutation are achieved by composing the canonical finite element interpolation operators with some mollification technique. Then, following Schöberl [29], the projection property over finite element spaces is obtained by composing these operators with the inverse of their restriction to the said spaces. An important extension of this construction allowing the possibility of using shape-regular mesh sequences and boundary conditions has been achieved by Christiansen and Winther [12]. Further variants of this construction have recently been proposed. For instance in Christiansen [11], the bounded cochain has the additional property of preserving polynomials locally, up to a certain degree, and in Falk and Winther [17] it is defined locally. In the present work, we revisit the results of [12] by invoking our shrinking-based mollification operators which do not require extension outside the domain. We also present the results in the language of numerical analysis to make them accessible to a wide audience. The main result of this second part is Theorem 6.4. As an application, we give in Theorem 6.5 discrete Poincaré inequalities for vector-valued functions.

The paper is organized as follows. In $\S 2$ we introduce a shrinking technique of $D$ that avoids difficulties with the boundary. We also introduce an expansion technique. In $\S 3$ we use the shrinking technique to devise mollification operators that commute with differential operators, are stable in any $L^{p}$ space, and have approximation properties on smooth functions. Using the expansion technique from $\S 2$, we introduce in $\S 4$ mollification operators that produce compactly supported functions and share the same properties as the shrinking-based operators. In $\S 5$, we introduce the finite element setting that is necessary to construct canonical interpolation operators on standard $H^{1}-, \boldsymbol{H}$ (curl)-, and $\boldsymbol{H}$ (div)-conforming finite element spaces, with and without homogeneous boundary conditions. In $\S 6$ we devise quasi-interpolant operators that are $L^{p}$-stable, commute with differential operators, and preserve finite element spaces, with and without boundary conditions.

2. Some geometry. In the entire paper, $D$ is an open, bounded, strongly Lipschitz, connected set in $\mathbb{R}^{d}$, int $(D)$ denotes the interior of $D$, and $\bar{D}$ its closure. Points in $\mathbb{R}^{d}$ and $\mathbb{R}^{d}$-valued functions and mappings are denoted using bold face; the Euclidean norm in $\mathbb{R}^{q}, q \geq 1$, is denoted $\|\cdot\|_{\ell^{2}\left(\mathbb{R}^{q}\right)}$, or $\|\cdot\|_{\ell^{2}}$ when the context is unam- 
biguous. We abuse the notation by using the same symbol for the $\ell^{2}$-induced matrix norm and the norm of multilinear maps.

2.1. Shrinking of strongly Lipschitz domains. Let $D$ be a strongly Lipschitz domain, i.e., there are $\alpha>0, \beta>0$, a finite number $N$ of affine maps $\boldsymbol{T}_{n}, n \in\{1: N\}$, and Lipschitz maps $\Phi_{n}: B\left(\mathbf{0}_{\mathbb{R}^{d-1}}, \alpha\right) \longrightarrow \mathbb{R}$ such that $\partial D=\bigcup_{n=1}^{N} \boldsymbol{T}_{n}(\{(\boldsymbol{x}, z=$ $\left.\left.\left.\Phi_{n}(\boldsymbol{x})\right) \mid \boldsymbol{x} \in B\left(\mathbf{0}_{\mathbb{R}^{d-1}}, \alpha\right)\right\}\right)$, and for all $n \in\{1: N\}$,

$$
\begin{aligned}
& \boldsymbol{T}_{n}\left(\left\{(\boldsymbol{x}, z) \mid \boldsymbol{x} \in B\left(\mathbf{0}_{\mathbb{R}^{d-1}}, \alpha\right), \Phi_{n}(\boldsymbol{x})<z<\Phi_{n}(\boldsymbol{x})+\beta\right\}\right) \subset \operatorname{int}(D), \\
& \boldsymbol{T}_{n}\left(\left\{(\boldsymbol{x}, z) \mid \boldsymbol{x} \in B\left(\mathbf{0}_{\mathbb{R}^{d-1}}, \alpha\right), \Phi_{n}(\boldsymbol{x})-\beta<z<\Phi_{n}(\boldsymbol{x})\right\}\right) \subset \mathbb{R}^{d} \backslash \bar{D},
\end{aligned}
$$

where $B\left(\mathbf{0}_{\mathbb{R}^{d-1}}, \alpha\right)$ is the open ball of radius $\alpha$ in $\mathbb{R}^{d-1}$ centered at the origin.

Since $D$ is strongly Lipschitz and bounded, the combination of Theorem 2.7 and Lemma 2.2 from Hofmann et al. [22] implies that $D$ has continuous globally transversal vector fields, i.e., there exist a vector field $\boldsymbol{J} \in \boldsymbol{C}^{0}(\partial D)$ and a real number $\gamma>0$ with the property that $\boldsymbol{n}(\boldsymbol{x}) \cdot \boldsymbol{J}(\boldsymbol{x}) \geq \gamma$ at a.e. point $\boldsymbol{x}$ on $\partial D$, where $\boldsymbol{n}$ is the unit normal vector pointing outward. Proposition 2.3 in [22] in turn implies the existence of a vector field $j \in C^{\infty}\left(\mathbb{R}^{d}\right)$ whose restriction to $\partial D$ is globally transversal and $\|\boldsymbol{j}(\boldsymbol{x})\|_{\ell^{2}}=1$ for all $\boldsymbol{x} \in \partial D$. We then define the mapping:

$$
\boldsymbol{\varphi}_{\delta}: \mathbb{R}^{d} \ni \boldsymbol{x} \mapsto \boldsymbol{x}-\delta \boldsymbol{j}(\boldsymbol{x}) \in \mathbb{R}^{d} .
$$

Using Proposition 4.15 in [22], together with the uniform cone property (see [22, pp. 599-600]), we infer that there exists $r>0$ such that

$$
\boldsymbol{\varphi}_{\delta}(D)+B(\mathbf{0}, \delta r) \subset D, \quad \forall \delta \in[0,1] .
$$

Lemma 2.1 (Properties of $\varphi_{\delta}$ ). The following properties hold:

(i) The map $\varphi_{\delta}$ is of class $C^{\infty}$ for all $\delta \in[0,1]$.

(ii) For all $\ell \in \mathbb{N}$, there is $c$ such that $\max _{\boldsymbol{x} \in D}\left\|D^{\ell} \boldsymbol{\varphi}_{\delta}(\boldsymbol{x})-D^{\ell} \boldsymbol{x}\right\|_{\ell^{2}} \leq c \delta$, for all $\delta \in[0,1]$, where $D^{\ell}$ denotes the Fréchet derivative of order $\ell$.

(iii) $\boldsymbol{\varphi}_{\delta}(D)+B(\mathbf{0}, \delta r) \subset D$ for all $\delta \in(0,1]$.

(iv) The mapping $\boldsymbol{x} \mapsto \boldsymbol{x}+t\left(\boldsymbol{\varphi}_{\delta}(\boldsymbol{x})+(\delta r \boldsymbol{y})-\boldsymbol{x}\right)$ maps $D$ into $D$ for all $t \in[0,1]$, all $\boldsymbol{y} \in B(\mathbf{0}, 1)$ and all $\delta \in[0,1]$.

Proof. The first two properties are consequences of $\boldsymbol{j}$ being of class $C^{\infty}$ and $D$ being bounded, while (iii) is just (2.2). To prove (iv), observe that $t\left(\boldsymbol{\varphi}_{\delta}(\boldsymbol{x})-\boldsymbol{x}\right)=$ $\boldsymbol{\varphi}_{t \delta}(\boldsymbol{x})-\boldsymbol{x}$. This implies that $\boldsymbol{x}+t\left(\boldsymbol{\varphi}_{\delta}(\boldsymbol{x})+(\delta r \boldsymbol{y})-\boldsymbol{x}\right)=\boldsymbol{\varphi}_{t \delta}(\boldsymbol{x})+t \delta r \boldsymbol{y} \in \boldsymbol{\varphi}_{t \delta}(D)+$ $B(\mathbf{0}, t \delta r) \subset D$ for all $\boldsymbol{y} \in B(\mathbf{0}, 1)$, all $t \in[0,1]$ and all $\delta \in[0,1]$.

2.2. Expansion of strongly Lipschitz domains. Since $D$ is bounded, there are $\boldsymbol{x}_{D} \in \mathbb{R}^{d}$ and $r_{D}>0$ such that $D \subset B\left(\boldsymbol{x}_{D}, r_{D}\right)$. Let $\mathcal{O}=B\left(\boldsymbol{x}_{D}, r_{D}\right) \backslash \bar{D}$. The domain $\mathcal{O}$ is bounded, open, and strongly Lipschitz; hence, we can apply the above argument again, and deduce the existence of a vector field $k \in C^{\infty}\left(\mathbb{R}^{d}\right)$ that is globally transversal for $\mathcal{O}$, points outward $D$, and $\|\boldsymbol{k}(\boldsymbol{x})\|_{\ell^{2}}=1$ for all $\boldsymbol{x} \in \partial O$; note that $\partial D \subset \partial O$. We then define the mapping:

$$
\boldsymbol{\vartheta}_{\delta}: \mathbb{R}^{d} \ni \boldsymbol{x} \longmapsto \boldsymbol{x}+\delta \boldsymbol{k}(\boldsymbol{x}) \in \mathbb{R}^{d} .
$$

As above, we infer that there exists $\zeta>0$ such that

$$
\boldsymbol{\vartheta}_{\delta}(\mathcal{O})+B(\mathbf{0}, 3 \delta \zeta) \subset \mathcal{O}, \quad \forall \delta \in[0,1] .
$$

Lemma 2.2 (Properties of $\boldsymbol{\vartheta}_{\delta}$ ). The following properties hold: 
(i) The map $\boldsymbol{\vartheta}_{\delta}$ is of class $C^{\infty}$ for all $\delta \in[0,1]$.

(ii) For all $\ell \in \mathbb{N}$, there is $c$ such that $\max _{\boldsymbol{x} \in D}\left\|D^{\ell} \boldsymbol{\vartheta}_{\delta}(\boldsymbol{x})-D^{\ell} \boldsymbol{x}\right\|_{\ell^{2}} \leq c \delta$, for all $\delta \in[0,1]$.

(iii) $\boldsymbol{\vartheta}_{\delta}(\overline{\mathcal{O}})+B(\mathbf{0}, 2 \delta \zeta) \subset \mathcal{O}$ for all $\delta \in(0,1]$.

Proof. The only novelty is the proof of (iii). Let $\boldsymbol{x} \in \overline{\mathcal{O}}$, then there exists $\boldsymbol{z}_{\delta} \in \mathcal{O}$ such that $\left(1+\delta L_{\boldsymbol{k}}\right)\left\|\boldsymbol{z}_{\delta}-\boldsymbol{x}\right\|_{\ell^{2}}<\delta \zeta$, where $L_{\boldsymbol{k}}$ denotes the Lipschitz constant of the field $\boldsymbol{k}$ in $\overline{\mathcal{O}}$. We observe that $\boldsymbol{\vartheta}_{\delta}(\boldsymbol{x})+B(\mathbf{0}, 2 \delta \zeta)=\boldsymbol{\vartheta}_{\delta}\left(\boldsymbol{z}_{\delta}\right)+\left(\boldsymbol{\vartheta}_{\delta}(\boldsymbol{x})-\boldsymbol{\vartheta}_{\delta}\left(\boldsymbol{z}_{\delta}\right)\right)+B(\mathbf{0}, 2 \delta \zeta) \subset$ $\boldsymbol{\vartheta}_{\delta}(\mathcal{O})+B(\mathbf{0}, \delta \zeta)+B(\mathbf{0}, 2 \delta \zeta)=\boldsymbol{\vartheta}_{\delta}(\mathcal{O})+B(\mathbf{0}, 3 \delta \zeta) \subset \mathcal{O}$ owing to (2.4).

3. Mollification without extension. We introduce in this section a mollification technique in strongly Lipschitz domains that does not require to invoke non-trivial extensions and that commutes with differential operators. The mapping $\varphi_{\delta}: D \longrightarrow D$ and $r>0$ are defined in (2.1) and (2.2). In what follows, $\mathbb{J}_{\delta}(\boldsymbol{x})$ denotes the Jacobian matrix of $\varphi_{\delta}$ at $\boldsymbol{x} \in D$.

3.1. Mollification. Let us consider the following kernel

$$
\rho(\boldsymbol{y}):= \begin{cases}\eta \exp \left(-\frac{1}{1-\|\boldsymbol{y}\|_{\ell^{2}}^{2}}\right), & \text { if }\|\boldsymbol{y}\|_{\ell^{2}}<1 \\ 0, & \text { if }\|\boldsymbol{y}\|_{\ell^{2}} \geq 1\end{cases}
$$

where $\eta$ is chosen so that $\int_{\mathbb{R}^{d}} \rho(\boldsymbol{y}) \mathrm{d} y=\int_{B(\mathbf{0}, 1)} \rho(\boldsymbol{y}) \mathrm{d} y=1$. To be generic, we

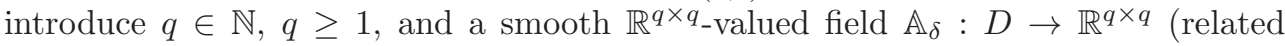
to the Jacobian $\mathbb{J}_{\delta}$ of $\varphi_{\delta}$, see (3.5) below) such that for all $l \in \mathbb{N}$, there is $c$ such that

$$
\sup _{\boldsymbol{x} \in D}\left\|D^{l}\left(\mathbb{A}_{\delta}(\boldsymbol{x})-\mathbb{I}\right)\right\|_{\ell^{2}} \leq c \delta
$$

where $\mathbb{I}$ is the identity matrix in $\mathbb{R}^{q \times q}$. Consider the following smoothing operators acting on $f \in L^{1}(D ; \mathbb{R})$ and $g=\left(g_{1}, \ldots, g_{q}\right)^{\top} \in L^{1}\left(D ; \mathbb{R}^{q}\right)$ :

$$
\begin{aligned}
&\left(\mathcal{K}_{\delta}^{\mathrm{g}} f\right)(\boldsymbol{x}):=\int_{B(\mathbf{0}, 1)} \rho(\boldsymbol{y}) f\left(\boldsymbol{\varphi}_{\delta}(\boldsymbol{x})+(\delta r) \boldsymbol{y}\right) \mathrm{d} y, \\
&\left(\mathcal{K}_{\delta} g\right)(\boldsymbol{x}):=\mathbb{A}_{\delta}(\boldsymbol{x})\left(\mathcal{K}_{\delta}^{\mathrm{g}} g_{1}(\boldsymbol{x}), \ldots, \mathcal{K}_{\delta}^{\mathrm{g}} g_{q}(\boldsymbol{x})\right)^{\top} .
\end{aligned}
$$

Property (iii) from Lemma 2.1 implies that

$$
\boldsymbol{\varphi}_{\delta}(\boldsymbol{x})+(\delta r) \boldsymbol{y} \in \boldsymbol{\varphi}_{\delta}(D)+(\delta r) B(\mathbf{0}, 1) \subset D, \quad \forall \boldsymbol{x} \in D, \forall \boldsymbol{y} \in B(\mathbf{0}, 1) .
$$

The means that the domains of $\mathcal{K}_{\delta}^{\mathrm{g}}$ and $\mathcal{K}_{\delta}$ are indeed $L^{1}(D ; \mathbb{R})$ and $L^{1}\left(D ; \mathbb{R}^{q}\right)$, i.e., there is no need to invoke extensions outside $D$.

Lemma 3.1 (Smoothness). $\mathcal{K}_{\delta} g$ is in $C^{\infty}\left(D ; \mathbb{R}^{q}\right)$ for all $g \in L^{1}\left(D ; \mathbb{R}^{q}\right)$, and $\mathcal{K}_{\delta} g$ as well as all its derivatives admit a continuous extension to $\bar{D}$.

Proof. Owing to (3.2) and (3.3b), and using the Leibniz product rule, it suffices to show that the statement holds for $\mathcal{K}_{\delta}^{\mathrm{g}}$. Let $f \in L^{1}(D)$. Let us prove first that $\mathcal{K}_{\delta}^{\mathrm{g}} f$ is continuous. Let $\boldsymbol{x}$ and $\boldsymbol{z}$ be two points in $D$. Up to appropriate changes of variable we have

$$
\mathcal{K}_{\delta}^{\mathrm{g}} f(\boldsymbol{x})-\mathcal{K}_{\delta}^{\mathrm{g}} f(\boldsymbol{z})=\frac{1}{(\delta r)^{d}} \int_{D}\left(\rho\left(\frac{\boldsymbol{y}-\boldsymbol{\varphi}_{\delta}(\boldsymbol{x})}{\delta r}\right)-\rho\left(\frac{\boldsymbol{y}-\boldsymbol{\varphi}_{\delta}(\boldsymbol{z})}{\delta r}\right)\right) f(\boldsymbol{y}) \mathrm{d} y,
$$

where we replaced $\boldsymbol{\varphi}_{\delta}(\boldsymbol{x})+(\delta r) B(\mathbf{0}, 1)$ and $\boldsymbol{\varphi}_{\delta}(\boldsymbol{z})+(\delta r) B(\mathbf{0}, 1)$ by $D$ and used that $\rho$ is zero outside the unit ball $B(\mathbf{0}, 1)$. The uniform Lipschitz continuity of $\rho$ and $\boldsymbol{\varphi}_{\delta}$ 
implies that there is $c$ such that $\left|\rho\left(\frac{\boldsymbol{y}-\boldsymbol{\varphi}_{\delta}(\boldsymbol{x})}{\delta r}\right)-\rho\left(\frac{\boldsymbol{y}-\boldsymbol{\varphi}_{\delta}(\boldsymbol{z})}{\delta r}\right)\right| \leq \frac{c}{\delta r}\|\boldsymbol{x}-\boldsymbol{z}\|_{\ell^{2}}$. As a result, we infer that

$$
\left|\mathcal{K}_{\delta}^{\mathrm{g}} f(\boldsymbol{x})-\mathcal{K}_{\delta}^{\mathrm{g}} f(\boldsymbol{z})\right| \leq c(\delta r)^{-d-1}\|f\|_{L^{1}(D)}\|\boldsymbol{x}-\boldsymbol{z}\|_{\ell^{2}},
$$

which proves that $\mathcal{K}_{\delta}^{\mathrm{g}} f$ is Lipschitz continuous; hence $\mathcal{K}_{\delta}^{\mathrm{g}} f$ is uniformly continuous. This proves that $\mathcal{K}_{\delta}^{\mathrm{g}} f \in C^{0}(D ; \mathbb{R})$ and $\mathcal{K}_{\delta}^{\mathrm{g}} f$ admits a continuous extension to $\bar{D}$. Let us now evaluate the gradient of $\mathcal{K}_{\delta}^{\mathrm{g}} f$. Using the chain rule, we infer that

$$
\begin{aligned}
\nabla\left(\mathcal{K}_{\delta}^{\mathrm{g}} f\right)(\boldsymbol{x}) & =\int_{B(\mathbf{0}, 1)} \rho(\boldsymbol{y}) \mathbb{J}_{\delta}^{\mathrm{T}}(\boldsymbol{x})(\nabla f)\left(\boldsymbol{\varphi}_{\delta}(\boldsymbol{x})+(\delta r) \boldsymbol{y}\right) \mathrm{d} y \\
& =\mathbb{J}_{\delta}^{\mathrm{\top}}(\boldsymbol{x}) \int_{B(\mathbf{0}, 1)} \rho(\boldsymbol{y})(\nabla f)\left(\boldsymbol{\varphi}_{\delta}(\boldsymbol{x})+(\delta r) \boldsymbol{y}\right) \mathrm{d} y \\
& =\mathbb{J}_{\delta}^{\mathrm{T}}(\boldsymbol{x})(\delta r)^{-1} \int_{B(\mathbf{0}, 1)} \rho(\boldsymbol{y}) \nabla\left(f\left(\boldsymbol{\varphi}_{\delta}(\boldsymbol{x})+(\delta r) \boldsymbol{y}\right)\right) \mathrm{d} y \\
& =-\mathbb{J}_{\delta}^{\mathrm{T}}(\boldsymbol{x})(\delta r)^{-1} \int_{B(\mathbf{0}, 1)} \nabla \rho(\boldsymbol{y}) f\left(\boldsymbol{\varphi}_{\delta}(\boldsymbol{x})+(\delta r) \boldsymbol{y}\right) \mathrm{d} y .
\end{aligned}
$$

We can then conclude that $\nabla\left(\mathcal{K}_{\delta}^{\mathrm{g}} f\right)$ is Lipschitz continuous by using the same argument as above and continue the argument by induction.

3.2. Examples. Let $f \in L^{1}(D ; \mathbb{R})$ and $\boldsymbol{g} \in L^{1}\left(D ; \mathbb{R}^{d}\right)$. Following [28] and [30, $\S 3]$, we define the following families of mollification operators:

$$
\begin{aligned}
& \left(\mathcal{K}_{\delta}^{\mathrm{g}} f\right)(\boldsymbol{x})=\int_{B(\mathbf{0}, 1)} \rho(\boldsymbol{y}) f\left(\boldsymbol{\varphi}_{\delta}(\boldsymbol{x})+(\delta r) \boldsymbol{y}\right) \mathrm{d} y, \\
& \left(\mathcal{K}_{\delta}^{\mathrm{c}} \boldsymbol{g}\right)(\boldsymbol{x})=\int_{B(\mathbf{0}, 1)} \rho(\boldsymbol{y}) \mathrm{J}_{\delta}^{\mathrm{T}}(\boldsymbol{x}) \boldsymbol{g}\left(\boldsymbol{\varphi}_{\delta}(\boldsymbol{x})+(\delta r) \boldsymbol{y}\right) \mathrm{d} y, \\
& \left(\mathcal{K}_{\delta}^{\mathrm{d}} \boldsymbol{g}\right)(\boldsymbol{x})=\int_{B(\mathbf{0}, 1)} \rho(\boldsymbol{y}) \operatorname{det}\left(\mathbb{J}_{\delta}(\boldsymbol{x})\right) \mathbb{J}_{\delta}^{-1}(\boldsymbol{x}) \boldsymbol{g}\left(\boldsymbol{\varphi}_{\delta}(\boldsymbol{x})+(\delta r) \boldsymbol{y}\right) \mathrm{d} y, \\
& \left(\mathcal{K}_{\delta}^{\mathrm{b}} f\right)(\boldsymbol{x})=\int_{B(\mathbf{0}, 1)} \rho(\boldsymbol{y}) \operatorname{det}\left(\mathbb{J}_{\delta}(\boldsymbol{x})\right) f\left(\boldsymbol{\varphi}_{\delta}(\boldsymbol{x})+(\delta r) \boldsymbol{y}\right) \mathrm{d} y,
\end{aligned}
$$

for all $\boldsymbol{x} \in D$. The superscripts in (3.4) refer to the fact that these operators are used to build projections onto finite element spaces that are conforming in the graph space of the gradient, curl, or divergence operator, or onto a broken finite element space (with no conformity requirement), see Theorem 6.4 below. The transformations involving $\mathbb{J}_{\delta}$ are related to the classical Piola transformations. Furthermore, the functions $\mathcal{K}_{\delta}^{\mathrm{c}} \boldsymbol{g}, \mathcal{K}_{\delta}^{\mathrm{d}} \boldsymbol{g}, \mathcal{K}_{\delta}^{\mathrm{b}} f$ are of the form (3.3b) with $\mathbb{A}_{\delta}^{\mathrm{g}}(\boldsymbol{x})=1$ and

$$
\mathbb{A}_{\delta}^{\mathrm{c}}(\boldsymbol{x})=\mathbb{J}_{\delta}^{\mathrm{T}}(\boldsymbol{x}), \quad \mathbb{A}_{\delta}^{\mathrm{d}}(\boldsymbol{x})=\operatorname{det}\left(\mathbb{J}_{\delta}(\boldsymbol{x})\right) \mathbb{J}_{\delta}^{-1}(\boldsymbol{x}), \quad \mathbb{A}_{\delta}^{\mathrm{b}}(\boldsymbol{x})=\operatorname{det}\left(\mathbb{J}_{\delta}(\boldsymbol{x})\right)
$$

Property (iii) from Lemma 2.1 implies that (3.2) holds true in the above three cases. Let $p \in[1, \infty]$. Assuming $d=3$, we define

$$
\begin{aligned}
& Z^{\mathrm{g}, p}(D)=\left\{f \in L^{p}(D) \mid \nabla f \in \boldsymbol{L}^{p}(D)\right\}, \\
& \boldsymbol{Z}^{\mathrm{c}, p}(D)=\left\{\boldsymbol{v} \in \boldsymbol{L}^{p}(D) \mid \nabla \times \boldsymbol{v} \in \boldsymbol{L}^{p}(D)\right\}, \\
& \boldsymbol{Z}^{\mathrm{d}, p}(D)=\left\{\boldsymbol{v} \in \boldsymbol{L}^{p}(D) \mid \nabla \cdot \boldsymbol{v} \in L^{p}(D)\right\} .
\end{aligned}
$$

Lemma 3.2 (Commuting with differential operators). The following holds: 
(i) $\nabla \mathcal{K}_{\delta}^{\mathrm{g}} f=\mathcal{K}_{\delta}^{\mathrm{c}} \nabla f$, for all $f \in Z^{\mathrm{g}, p}(D)$,

(ii) $\nabla \times \mathcal{K}_{\delta}^{\mathrm{c}} \boldsymbol{g}=\mathcal{K}_{\delta}^{\mathrm{d}} \nabla \times \boldsymbol{g}$, for all $\boldsymbol{g} \in \boldsymbol{Z}^{\mathrm{c}, p}(D)$,

(iii) $\nabla \cdot \mathcal{K}_{\delta}^{\mathrm{d}} \boldsymbol{g}=\mathcal{K}_{\delta}^{\mathrm{b}} \nabla \cdot \boldsymbol{g}$, for all $\boldsymbol{g} \in \boldsymbol{Z}^{\mathrm{d}, p}(D)$,

i.e., the following diagram commutes:

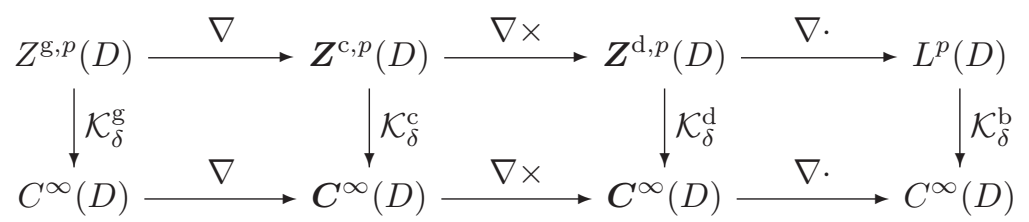

Proof. Upon setting $\boldsymbol{T}(\boldsymbol{x})=\boldsymbol{\varphi}_{\delta}(\boldsymbol{x})+\delta r \boldsymbol{y}$, these identities are simple consequences of the chain rule:

$$
\begin{aligned}
\nabla(f \circ \boldsymbol{T})(\boldsymbol{x}) & =\mathbb{J}_{\delta}^{\top}(\boldsymbol{x})(\nabla f)(\boldsymbol{T}(\boldsymbol{x})), \\
\nabla \times\left(\mathbb{J}_{\delta}^{\top}(\boldsymbol{x})(\boldsymbol{g} \circ \boldsymbol{T})\right)(\boldsymbol{x}) & =\operatorname{det}\left(\mathbb{J}_{\delta}(\boldsymbol{x})\right) \mathbb{J}_{K}^{-1}(\boldsymbol{x})(\nabla \times \boldsymbol{g})(\boldsymbol{T}(\boldsymbol{x})), \\
\nabla \cdot\left(\operatorname{det}\left(\mathbb{J}_{\delta}(\boldsymbol{x})\right) \mathbb{J}_{\delta}^{-1}(\boldsymbol{g} \circ \boldsymbol{T})\right)(\boldsymbol{x}) & =\operatorname{det}\left(\mathbb{J}_{\delta}(\boldsymbol{x})\right)(\nabla \cdot \boldsymbol{g})(\boldsymbol{T}(\boldsymbol{x})) .
\end{aligned}
$$

This completes the proof.

3.3. Convergence. We now show that the smoothing operators defined above have interesting approximation properties. Owing to Lemma 2.1, $\mathbb{J}_{\delta}$ and $\mathbb{J}_{\delta}^{-1}$ converge uniformly to the identity, and $\operatorname{det}\left(\mathbb{J}_{\delta}\right)$ converges uniformly to 1 . As a result, there is $\delta_{0} \in(0,1]$ such that $\left\|\mathbb{J}_{\delta}-\mathbb{I}\right\|_{\ell^{2}} \leq \frac{1}{2},\left\|\mathbb{J}_{\delta}^{-1}\right\|_{\ell^{2}} \leq 2$, and $\left|\operatorname{det}\left(\mathbb{J}_{\delta}^{-1}\right)\right| \leq 2^{d}$, for all $\delta \in\left[0, \delta_{0}\right]$ and all $\boldsymbol{x} \in D$.

THEOREM 3.3 (Convergence). The sequence $\left(\mathcal{K}_{\delta}\right)_{\delta \in\left[0, \delta_{0}\right]}$ is uniformly bounded in $\mathcal{L}\left(L^{p} ; L^{p}\right):=\mathcal{L}\left(L^{p}\left(D ; \mathbb{R}^{q}\right) ; L^{p}\left(D ; \mathbb{R}^{q}\right)\right)$ for all $p \in[0, \infty]$. Moreover, for $p \in[1, \infty)$, $\left\|\mathcal{K}_{\delta} f-f\right\|_{L^{p}\left(D ; \mathbb{R}^{q}\right)} \rightarrow 0$ as $\delta \rightarrow 0$ for all $f \in L^{p}\left(D ; \mathbb{R}^{q}\right)$.

Proof. Owing to (3.2) and (3.3b), it suffices to show that the statement holds for $\mathcal{K}_{\delta}^{\mathrm{g}}$. (1) We show first that $\mathcal{K}_{\delta}^{\mathrm{g}}$ is uniformly bounded in $\mathcal{L}\left(L^{p} ; L^{p}\right)$ by using the RieszThorin interpolation theorem. The statement is evident for $p=\infty$ with constant $c=1$. Now consider $f \in L^{1}(D ; \mathbb{R})$, then

$$
\begin{aligned}
\left\|\mathcal{K}_{\delta}^{\mathrm{g}} f\right\|_{L^{1}(D)} & \leq \int_{D} \int_{B(\mathbf{0}, 1)} \rho(\boldsymbol{y})\left|f\left(\boldsymbol{\varphi}_{\delta}(\boldsymbol{x})+(\delta r) \boldsymbol{y}\right)\right| \mathrm{d} y \mathrm{~d} x \\
& \leq \int_{B(\mathbf{0}, 1)} \rho(\boldsymbol{y}) \int_{D}\left|f\left(\boldsymbol{\varphi}_{\delta}(\boldsymbol{x})+(\delta r) \boldsymbol{y}\right)\right| \mathrm{d} x \mathrm{~d} y \\
& \leq \int_{B(\mathbf{0}, 1)} \rho(\boldsymbol{y}) \int_{\boldsymbol{\varphi}_{\delta}(D)+(\delta r) \boldsymbol{y}}\left|f(\boldsymbol{z})\left\|\left.\operatorname{det}\left(\mathbb{J}_{\delta}(\boldsymbol{z})\right)\right|^{-1} \mathrm{~d} z \mathrm{~d} y \leq c\right\| f \|_{L^{1}(D)},\right.
\end{aligned}
$$

since $\delta \leq \delta_{0}$. The Riesz-Thorin interpolation theorem implies that $\left\|\mathcal{K}_{\delta}^{\mathrm{g}} f\right\|_{L^{p}(D)} \leq$ $c^{\frac{1}{p}}\|f\|_{L^{p}(D)}$, so that $\left\|\mathcal{K}_{\delta}^{\mathrm{g}} f\right\|_{L^{p}(D)} \leq c_{1}\|f\|_{L^{p}(D)}$, with $c_{1}=\max (1, c)$.

(2) Assume first that $f$ is smooth over $D$, say uniformly Lipschitz with Lipschitz constant $L_{f}$, i.e., $|f(\boldsymbol{x})-f(\boldsymbol{z})| \leq L_{f}\|\boldsymbol{x}-\boldsymbol{z}\|_{\ell^{2}}$. Then,

$$
\begin{aligned}
\left|\mathcal{K}_{\delta}^{\mathrm{g}} f(\boldsymbol{x})-f(\boldsymbol{x})\right| & =\left|\int_{B(\mathbf{0}, 1)} \rho(\boldsymbol{y})\left(f\left(\boldsymbol{\varphi}_{\delta}(\boldsymbol{x})+(\delta r) \boldsymbol{y}\right)-f(\boldsymbol{x})\right) \mathrm{d} y\right| \\
& \left.\leq \int_{B(\mathbf{0}, 1)} \rho(\boldsymbol{y}) L_{f} \| \boldsymbol{\varphi}_{\delta}(\boldsymbol{x})-\boldsymbol{x}+(\delta r) \boldsymbol{y}\right) \|_{\ell^{2}} \mathrm{~d} y \leq c L_{f} \delta .
\end{aligned}
$$


In conclusion, there is $c_{0}=c \max (1,|D|)$ such that $\left\|\mathcal{K}_{\delta}^{\mathrm{g}} f-f\right\|_{L^{p}(D)} \leq c_{0} L_{f} \delta$.

(3) We conclude by using a density argument and the triangle inequality. Let $f \in$ $L^{p}(D)$. The space of uniformly Lipschitz functions being dense in $L^{p}(D)$, there is a sequence of uniformly Lipschitz functions $\left(f_{n}\right)_{n \in \mathbb{N}}$ such that $\left\|f_{n}-f\right\|_{L^{p}(D)} \rightarrow 0$ as $n \rightarrow \infty$. Then

$$
\begin{aligned}
\left\|\mathcal{K}_{\delta}^{\mathrm{g}} f-f\right\|_{L^{p}(D)} & \leq\left\|\mathcal{K}_{\delta}^{\mathrm{g}}\left(f-f_{n}\right)\right\|_{L^{p}(D)}+\left\|\mathcal{K}_{\delta}^{\mathrm{g}} f_{n}-f_{n}\right\|_{L^{p}(D)}+\left\|f_{n}-f\right\|_{L^{p}(D)} \\
& \leq c_{1}\left\|f-f_{n}\right\|_{L^{p}(D)}+c_{0} L_{f_{n}} \delta+\left\|f_{n}-f\right\|_{L^{p}(D)} .
\end{aligned}
$$

Let $\epsilon>0$ and let $n(\epsilon)$ be large enough so that $\left\|f_{n(\epsilon)}-f\right\|_{L^{p}(D)} \leq \epsilon$. Setting $\delta_{0}(\epsilon)=$ $\epsilon / L_{f_{n(\epsilon)}}$, we have $\left\|\mathcal{K}_{\delta}^{\mathrm{g}} f-f\right\|_{L^{p}(D)} \leq \epsilon\left(c_{1}+c_{0}+1\right)$ for all $\delta \leq \delta_{0}(\epsilon)$. In conclusion, for all $\epsilon>0$, there is $\delta_{0}(\epsilon)$ such that $\left\|\mathcal{K}_{\delta}^{\mathrm{g}} f-f\right\|_{L^{p}(D)} \leq \epsilon\left(c_{1}+c_{0}+1\right)$ for all $\delta \leq \delta_{0}(\epsilon)$, which proves that $\left\|\mathcal{K}_{\delta}^{\mathrm{g}} f-f\right\|_{L^{p}(D)} \rightarrow 0$ as $\delta \rightarrow 0$.

We now can state a result that shows that the above smoothing technique is superior to mollification alone, i.e., contrary to the result originally stated by Friedrichs (see e.g., [8, Thm 9.2]), strong convergence on the derivatives now occurs over the entire domain $D$.

Corollary 3.4 (Convergence of derivatives). Let $p \in[1, \infty)$. Then,

$$
\begin{array}{cl}
\lim _{\delta \rightarrow 0}\left\|\nabla\left(\mathcal{K}_{\delta}^{\mathrm{g}} f-f\right)\right\|_{\boldsymbol{L}^{p}(D)}=0, & \forall f \in Z^{\mathrm{g}, p}(D), \\
\lim _{\delta \rightarrow 0}\left\|\nabla \times\left(\mathcal{K}_{\delta}^{\mathrm{c}} \boldsymbol{g}-\boldsymbol{g}\right)\right\|_{\boldsymbol{L}^{p}(D)}=0, & \forall \boldsymbol{g} \in \boldsymbol{Z}^{\mathrm{c}, p}(D), \\
\lim _{\delta \rightarrow 0}\left\|\nabla \cdot\left(\mathcal{K}_{\delta}^{\mathrm{d}} \boldsymbol{g}-\boldsymbol{g}\right)\right\|_{L^{p}(D)}=0, & \forall \boldsymbol{g} \in \boldsymbol{Z}^{\mathrm{d}, p}(D) .
\end{array}
$$

Proof. Using Lemma 3.2 we infer that $\nabla \mathcal{K}_{\delta}^{\mathrm{g}} f=\mathcal{K}_{\delta}^{\mathrm{c}} \nabla f$, and Theorem 3.3 implies that $\mathcal{K}_{\delta}^{c} \nabla f \rightarrow \nabla f$ in $\boldsymbol{L}^{p}(D)$ as $\delta \rightarrow 0$, which proves (3.8a). A similar argument holds for $(3.8 \mathrm{~b})$ and $(3.8 \mathrm{c})$.

3.4. Convergence rate. We now establish convergence rates.

Theorem 3.5 (Convergence rate). There is c such that

$$
\left\|\mathcal{K}_{\delta} f-f\right\|_{L^{p}\left(D ; \mathbb{R}^{q}\right)} \leq c \delta^{s}|f|_{W^{s, p}\left(D ; \mathbb{R}^{q}\right)},
$$

for all $f \in W^{s, p}\left(D ; \mathbb{R}^{q}\right)$, all $\delta \in\left[0, \delta_{0}\right]$, and all $s \in(0,1], p \in[1, \infty)$ or $s=1$, $p \in[1, \infty]$.

Proof. Owing to (3.2) and (3.3b), it suffices to show that the statement holds for $\mathcal{K}_{\delta}^{\mathrm{g}}$. Assume first that $p<\infty$.

(1) Let $f \in W^{s, p}(D)$ with $s \in(0,1)$. We estimate $\mathcal{K}_{\delta}^{\mathrm{g}} f-f$ in $L^{p}(D)$ as follows:

$$
\begin{aligned}
\| \mathcal{K}_{\delta}^{\mathrm{g}} f- & f \|_{L^{p}(D)}^{p}=\int_{D}\left|\int_{B(\mathbf{0}, 1)} \rho(y)\left(f\left(\boldsymbol{\varphi}_{\delta}(\boldsymbol{x})+(\delta r) \boldsymbol{y}\right)-f(\boldsymbol{x})\right) \mathrm{d} y\right|^{p} \mathrm{~d} x \\
& \leq c \int_{B(\mathbf{0}, 1)} \int_{D} \frac{\left|f\left(\boldsymbol{\varphi}_{\delta}(\boldsymbol{x})+(\delta r) \boldsymbol{y}\right)-f(\boldsymbol{x})\right|^{p}}{\left\|\boldsymbol{\varphi}_{\delta}(\boldsymbol{x})+(\delta r) \boldsymbol{y}-\boldsymbol{x}\right\|_{\ell^{2}}^{s p+d}}\left\|\boldsymbol{\varphi}_{\delta}(\boldsymbol{x})+(\delta r) \boldsymbol{y}-\boldsymbol{x}\right\|_{\ell^{2}}^{s p+d} \mathrm{~d} x \mathrm{~d} y .
\end{aligned}
$$

Let us make the change of variables $B(\mathbf{0}, 1) \ni \boldsymbol{y} \mapsto \boldsymbol{z}=\boldsymbol{\varphi}_{\delta}(\boldsymbol{x})+(\delta r) \boldsymbol{y} \in \boldsymbol{\varphi}_{\delta}(D)+$ $\delta r B(\mathbf{0}, 1) \subset D$. Observe that the Jacobian of this transformation is bounded from above by $\delta r$ and

$$
\left\|\boldsymbol{\varphi}_{\delta}(\boldsymbol{x})+(\delta r) \boldsymbol{y}-\boldsymbol{x}\right\|_{\ell^{2}} \leq\left\|\boldsymbol{\varphi}_{\delta}(\boldsymbol{x})-\boldsymbol{x}\right\|_{\ell^{2}}+\delta r\|\boldsymbol{y}\|_{\ell^{2}} \leq c \delta
$$


Hence,

$$
\left\|\mathcal{K}_{\delta}^{\mathrm{g}} f-f\right\|_{L^{p}(D)}^{p} \leq c \delta^{s p+d} \delta^{-d} \int_{D} \int_{D} \frac{|f(\boldsymbol{z})-f(\boldsymbol{x})|^{p}}{\|\boldsymbol{z}-\boldsymbol{x}\|_{\ell^{2}}^{s p+d}} \mathrm{~d} x \mathrm{~d} z \leq c \delta^{s p}|f|_{W^{s, p}(D)}^{p} .
$$

(2) Let $f \in W^{1, p}(D)$. By proceeding as above we infer that

$$
\left\|\mathcal{K}_{\delta}^{\mathrm{g}} f-f\right\|_{L^{p}(D)}^{p} \leq c \int_{B(\mathbf{0}, 1)} \int_{D}\left|f\left(\boldsymbol{\varphi}_{\delta}(\boldsymbol{x})+(\delta r) \boldsymbol{y}\right)-f(\boldsymbol{x})\right|^{p} \mathrm{~d} x \mathrm{~d} y .
$$

Let us fix $y \in B(\mathbf{0}, 1)$ and define the mapping $\boldsymbol{\psi}_{\delta}: D \ni \boldsymbol{x} \mapsto \boldsymbol{\varphi}_{\delta}(\boldsymbol{x})+(\delta r) \boldsymbol{y} \in$ $\boldsymbol{\varphi}_{\delta}(D)+\delta r B(\mathbf{0}, 1) \subset D$. Observe that

$\left\|\boldsymbol{\psi}_{\delta}(\boldsymbol{x})-\boldsymbol{x}\right\|_{\ell^{2}} \leq\left\|\boldsymbol{\varphi}_{\delta}(\boldsymbol{x})-\boldsymbol{x}\right\|_{\ell^{2}}+\delta r\|\boldsymbol{y}\|_{\ell^{2}} \leq c \delta, \quad\left\|D \boldsymbol{\psi}_{\delta}(\boldsymbol{x})-\mathbb{I}\right\|=\left\|D \boldsymbol{\varphi}_{\delta}(\boldsymbol{x})-\mathbb{I}\right\| \leq c \delta$, and $\boldsymbol{x}+t\left(\boldsymbol{\psi}_{\delta}(\boldsymbol{x})-\boldsymbol{x}\right)=\boldsymbol{x}+t\left(\boldsymbol{\varphi}_{\delta}(\boldsymbol{x})+\delta r \boldsymbol{y}-\boldsymbol{x}\right) \in D$, i.e., $\boldsymbol{\psi}_{\delta}$ satisfies the assumptions of Lemma 3.7 below. Hence, $\int_{D}\left|f\left(\boldsymbol{\varphi}_{\delta}(\boldsymbol{x})+(\delta r) \boldsymbol{y}\right)-f(\boldsymbol{x})\right|^{p} \mathrm{~d} x \leq c \delta^{p}\|\nabla f\|_{\boldsymbol{L}^{p}(D)}^{p}$. We conclude that $\left\|\mathcal{K}_{\delta}^{\mathrm{g}} f-f\right\|_{L^{p}(D)} \leq c \delta\|\nabla f\|_{\boldsymbol{L}^{p}(D)}$.

(3) The case $s=1, p=\infty$ is treated similarly to (2).

Corollary 3.6 (Convergence rate on derivatives). Let $s \in(0,1), p \in[1, \infty)$ or $s=1, p \in[1, \infty]$. Then, there is c such that

$$
\begin{aligned}
\left\|\nabla\left(\mathcal{K}_{\delta}^{\mathrm{g}} f-f\right)\right\|_{\boldsymbol{L}^{p}(D)} \leq c \delta^{s}|\nabla f|_{\boldsymbol{W}^{s, p}(D)}, & & \forall f \in\left\{v \in L^{p}(D) \mid \nabla v \in \boldsymbol{W}^{s, p}(D)\right\}, \\
\left\|\nabla \times\left(\mathcal{K}_{\delta}^{\mathrm{c}} \boldsymbol{g}-\boldsymbol{g}\right)\right\|_{\boldsymbol{L}^{p}(D)} \leq c \delta^{s}|\nabla \times \boldsymbol{g}|_{\boldsymbol{W}^{s, p}(D)}, & & \forall \boldsymbol{g} \in\left\{\boldsymbol{v} \in \boldsymbol{L}^{p}(D) \mid \nabla \times \boldsymbol{v} \in \boldsymbol{W}^{s, p}(D)\right\}, \\
\left\|\nabla \cdot\left(\mathcal{K}_{\delta}^{\mathrm{d}} \boldsymbol{g}-\boldsymbol{g}\right)\right\|_{L^{p}(D)} \leq c \delta^{s}|\nabla \cdot \boldsymbol{g}|_{W^{s, p}(D)}, & & \forall \boldsymbol{g} \in\left\{\boldsymbol{v} \in \boldsymbol{L}^{p}(D) \mid \nabla \cdot \boldsymbol{v} \in W^{s, p}(D)\right\} .
\end{aligned}
$$

Proof. Let $f \in\left\{v \in L^{p}(D) \mid \nabla v \in \boldsymbol{W}^{s, p}(D)\right\}$, then

$$
\begin{aligned}
\left\|\nabla\left(\mathcal{K}_{\delta}^{\mathrm{g}} f-f\right)\right\|_{\boldsymbol{L}^{p}(D)} & =\left\|\mathcal{K}_{\delta}^{\mathrm{c}} \nabla f-\nabla f\right\|_{\boldsymbol{L}^{p}(D)} & & \text { since } \nabla \mathcal{K}_{\delta}^{\mathrm{g}}=\mathcal{K}_{\delta}^{\mathrm{c}} \nabla \\
& \leq c \delta^{s}\|\nabla f\|_{\boldsymbol{W}^{s, p}(D)} & & \text { owing to Theorem 3.5. }
\end{aligned}
$$

Proceed similarly for the two other estimates. $\square$

LEMma 3.7 (Approximation). Let $\lambda_{0}>0$, and assume that $\boldsymbol{\psi}_{\lambda}: D \rightarrow D$ is a diffeomorphism of class $C^{1}$ such that $\left\|\boldsymbol{\psi}_{\lambda}(\boldsymbol{x})-\boldsymbol{x}\right\|_{\ell^{2}} \leq c^{\prime} \lambda$ and $\left\|D \boldsymbol{\psi}_{\lambda}(\boldsymbol{x})-\mathbb{I}\right\|_{\ell^{2}} \leq \frac{1}{2}$ for all $\boldsymbol{x} \in D$ and all $\lambda \in\left[0, \lambda_{0}\right]$. Assume also that the mapping $\boldsymbol{\mu}_{\lambda, t}: \boldsymbol{x} \mapsto \boldsymbol{x}+$ $t\left(\boldsymbol{\psi}_{\lambda}(\boldsymbol{x})-\boldsymbol{x}\right)$ maps $D$ into $D$ for all $t \in[0,1]$ and all $\lambda \in\left[0, \lambda_{0}\right]$. Then, there is $c$ such that the following holds:

$$
\left\|f \circ \boldsymbol{\psi}_{\lambda}-f\right\|_{L^{p}(D)} \leq c \lambda\|\nabla f\|_{\boldsymbol{L}^{p}(D)},
$$

for all $\lambda \in\left[0, \lambda_{0}\right]$, all $f \in W^{1, p}(D)$, and all $p \in[1, \infty]$.

Proof. (1) Assume first that $f$ is smooth. Let $\boldsymbol{x} \in D$ and $v(t):=f\left(\boldsymbol{\mu}_{\lambda, t}(\boldsymbol{x})\right)$ with $t \in[0,1]$. The chain rule implies that $v^{\prime}(t)=\operatorname{Df}\left(\boldsymbol{\mu}_{\lambda, t}(\boldsymbol{x})\right)\left(\boldsymbol{\psi}_{\lambda}(\boldsymbol{x})-\boldsymbol{x}\right)$, thereby showing that

$$
f\left(\boldsymbol{\psi}_{\lambda}(\boldsymbol{x})\right)-f(\boldsymbol{x})=\int_{0}^{1} v^{\prime}(t) \mathrm{d} t=\int_{0}^{1} D f\left(\boldsymbol{\mu}_{\lambda, t}(\boldsymbol{x})\right)\left(\boldsymbol{\psi}_{\lambda}(\boldsymbol{x})-\boldsymbol{x}\right) \mathrm{d} t
$$

Then, assuming that $p<\infty$, we infer that

$$
\begin{aligned}
\left\|f \circ \boldsymbol{\psi}_{\lambda}-f\right\|_{L^{p}(D)}^{p} & \leq \int_{D}\left\|\boldsymbol{\psi}_{\lambda}(\boldsymbol{x})-\boldsymbol{x}\right\|_{\ell^{2}}^{p} \int_{0}^{1}\left\|\nabla f\left(\boldsymbol{\mu}_{\lambda, t}(\boldsymbol{x})\right)\right\|_{\ell^{2}}^{p} \mathrm{~d} t \mathrm{~d} x \\
& \leq c^{\prime} \lambda^{p} \int_{0}^{1} \int_{D}\left\|\nabla f\left(\boldsymbol{\mu}_{\lambda, t}(\boldsymbol{x})\right)\right\|_{\ell^{2}}^{p} \mathrm{~d} t \mathrm{~d} x .
\end{aligned}
$$


The assumptions on $\boldsymbol{\psi}_{\lambda}$ imply that the map $\boldsymbol{\mu}_{\lambda, t}$ is invertible and $\left\|D \boldsymbol{\mu}_{\lambda, t}^{-1}\right\|_{\ell^{2}} \leq 2$, $\left|\operatorname{det}\left(D \boldsymbol{\mu}_{\lambda, t}^{-1}\right)\right| \leq 2^{d}$. As a result,

$$
\left\|f \circ \boldsymbol{\psi}_{\lambda}-f\right\|_{L^{p}(D)}^{p} \leq c^{\prime} \lambda^{p} \int_{0}^{1} \int_{D}\|\nabla f(\boldsymbol{z})\|_{\ell^{2}}^{p}\left|\operatorname{det}\left(D \boldsymbol{\mu}_{\lambda, t}^{-1}\right)\right| \mathrm{d} z \mathrm{~d} t,
$$

which finally implies that there is $c_{0}$ so that $\left\|f \circ \boldsymbol{\psi}_{\lambda}-f\right\|_{L^{p}(D)} \leq c_{0} \lambda\|\nabla f\|_{\boldsymbol{L}^{p}(D)}$. The case $p=\infty$ is treated similarly.

(2) If $f$ is not smooth, we deduce from Corollary 3.4 that there exists a sequence of smooth functions converging to $f$ in $W^{1, p}(D)$, i.e., for all $\epsilon>0$, there is a smooth function $f_{\epsilon}$ such that $\left\|f-f_{\epsilon}\right\|_{L^{p}(D)} \leq \epsilon$ and $\left\|\nabla f_{\epsilon}\right\|_{\boldsymbol{L}^{p}(D)} \leq 2\|\nabla f\|_{\boldsymbol{L}^{p}(D)}$. Then

$$
\begin{aligned}
\left\|f \circ \boldsymbol{\psi}_{\lambda}-f\right\|_{L^{p}(D)} & \leq\left\|\left(f-f_{\epsilon}\right) \circ \boldsymbol{\psi}_{\lambda}\right\|_{L^{p}(D)}+\left\|f_{\epsilon} \circ \boldsymbol{\psi}_{\lambda}-f_{\epsilon}\right\|_{L^{p}(D)}+\left\|f_{\epsilon}-f\right\|_{L^{p}(D)} \\
& \leq c \epsilon+2 c_{0} \lambda\|\nabla f\|_{L^{p}(D)}+\epsilon
\end{aligned}
$$

The conclusion follows readily since $\epsilon$ is arbitrary.

4. Mollification with extension by zero. Note that the function $\mathcal{K}_{\delta} f$ defined in $(3.3 \mathrm{~b})$ does not satisfy any particular boundary condition. For instance, even if $f$ is zero on $\partial D,\left(\mathcal{K}_{\delta} f\right)_{\mid \partial D}$ is not necessarily zero. Since preserving boundary conditions may be useful in some applications, we now construct a mollifier that has this property. Let $C_{0}^{\infty}\left(D ; \mathbb{R}^{q}\right)$ denote the space of $\mathbb{R}^{q}$-valued functions that are of class $C^{\infty}$ and of compact support in $D$. Consider the mapping $\boldsymbol{\vartheta}_{\delta}$ and the constant $\zeta$ defined in $(2.3)$ and (2.4). Let $\mathbb{K}_{\delta}(\boldsymbol{x})$ denote the Jacobian matrix of $\boldsymbol{\vartheta}_{\delta}$ at $\boldsymbol{x} \in D$.

4.1. Mollification. For any $g \in L^{1}\left(D ; \mathbb{R}^{q}\right), q \in \mathbb{N}$ with $q \geq 1$, we denote by $\widetilde{g}$ the extension by zero of $g$ over $\mathbb{R}^{d}$, i.e., $\widetilde{g}(\boldsymbol{x})=g(\boldsymbol{x})$ if $\boldsymbol{x} \in D$ and $\widetilde{g}(\boldsymbol{x})=0$ otherwise. Taking inspiration from Bonito et al. [7], we introduce

$$
\begin{aligned}
\left(\mathcal{K}_{\delta, 0}^{\mathrm{g}} f\right)(\boldsymbol{x}) & :=\int_{B(\mathbf{0}, 1)} \rho(\boldsymbol{y}) \widetilde{f}\left(\boldsymbol{\vartheta}_{\delta}(\boldsymbol{x})+(\delta \zeta) \boldsymbol{y}\right) \mathrm{d} y, \\
\left(\mathcal{K}_{\delta, 0} g\right)(\boldsymbol{x}): & =\mathbb{B}_{\delta}(\boldsymbol{x})\left(\mathcal{K}_{\delta, 0}^{\mathrm{g}} g_{1}(\boldsymbol{x}), \ldots, \mathcal{K}_{\delta, 0}^{\mathrm{g}} g_{q}(\boldsymbol{x})\right)^{\top},
\end{aligned}
$$

for all $\boldsymbol{x} \in D$, all $f \in L^{1}(D ; \mathbb{R})$, and all $g=\left(g_{1}, \ldots, g_{q}\right)^{\top} \in L^{1}\left(D ; \mathbb{R}^{q}\right)$, where $\mathbb{B}_{\delta}$ is a smooth $\mathbb{R}^{q \times q}$-valued field (related to the Jacobian $\mathbb{K}_{\delta}$ of $\boldsymbol{\vartheta}_{\delta}$ ) such that for all $l \in \mathbb{N}$, there is $c$ such that

$$
\sup _{\boldsymbol{x} \in D}\left\|D^{l}\left(\mathbb{B}_{\delta}(\boldsymbol{x})-\mathbb{I}\right)\right\|_{\ell^{2}} \leq c \delta
$$

LEMMA 4.1 (Smoothness and boundary condition). $\mathcal{K}_{\delta, 0}(g)$ is in $C_{0}^{\infty}\left(D ; \mathbb{R}^{q}\right)$ for all $g \in L^{1}\left(D ; \mathbb{R}^{q}\right)$ and all $\delta \in(0,1]$.

Proof. The smoothness has already been proved in Lemma 3.1. Let $\kappa$ be the Lipschitz constant of the field $\boldsymbol{k}$ over $\bar{D}$. Let $\epsilon_{\delta}=\delta \zeta /(1+\delta \kappa)$. Let $\boldsymbol{x} \in D$ be such that $\operatorname{dist}(\boldsymbol{x}, \partial D)<\epsilon_{\delta}$. Then, there exists a point $\boldsymbol{z} \in \partial D \operatorname{such}$ that $\operatorname{dist}(\boldsymbol{x}, \boldsymbol{z}) \leq \epsilon_{\delta}$, i.e.,

$$
\begin{aligned}
\boldsymbol{\vartheta}_{\delta}(\boldsymbol{x})+B(\mathbf{0}, \delta \zeta) & =\boldsymbol{\vartheta}_{\delta}(\boldsymbol{z})+B(\mathbf{0}, \delta \zeta)+\boldsymbol{\vartheta}_{\delta}(\boldsymbol{x})-\boldsymbol{\vartheta}_{\delta}(\boldsymbol{z}) \\
& =\boldsymbol{\vartheta}_{\delta}(\boldsymbol{z})+B(\mathbf{0}, \delta \zeta)+\boldsymbol{x}-\boldsymbol{z}+\delta(\boldsymbol{k}(\boldsymbol{x})-\boldsymbol{k}(\boldsymbol{z})) \\
& \subset \boldsymbol{\vartheta}_{\delta}(\boldsymbol{z})+B(\mathbf{0}, \delta \zeta)+B\left(\mathbf{0}, \epsilon_{\delta}+\delta \kappa \epsilon_{\delta}\right) \\
& =\boldsymbol{\vartheta}_{\delta}(\boldsymbol{z})+B\left(\mathbf{0}, \delta \zeta+(1+\delta \kappa) \epsilon_{\delta}\right)=\boldsymbol{\vartheta}_{\delta}(\boldsymbol{z})+B(\mathbf{0}, 2 \delta \zeta) \\
& \subset \boldsymbol{\vartheta}_{\delta}(\overline{\mathcal{O}})+B(\mathbf{0}, 2 \delta \zeta) \subset \mathcal{O},
\end{aligned}
$$


owing to Lemma 2.2(iii). This implies that $\boldsymbol{\vartheta}_{\delta}(\boldsymbol{x})+(\delta \zeta) \boldsymbol{y} \subset \mathcal{O}$ for all $\boldsymbol{y} \in B(\mathbf{0}, 1)$, so that $\left(\mathcal{K}_{\delta, 0}^{\mathrm{g}}(f)\right)(\boldsymbol{x})=0$ since $f\left(\boldsymbol{\vartheta}_{\delta}(\boldsymbol{x})+(\delta \zeta) \boldsymbol{y}\right)=0$ for all $\boldsymbol{y} \in B(\mathbf{0}, 1)$. Hence, the support of $\mathcal{K}_{\delta, 0}^{\mathrm{g}}$ is compact in $D$. The same conclusion applies to $\mathcal{K}_{\delta, 0}$.

4.2. Examples. Let us proceed as in $\S 3.2$. Let $\mathbb{B}_{\delta}^{\mathrm{c}}(\boldsymbol{x})=\mathbb{K}_{\delta}^{\top}(\boldsymbol{x}), \mathbb{B}_{\delta}^{\mathrm{d}}(\boldsymbol{x})=$ $\operatorname{det}\left(\mathbb{K}_{\delta}(\boldsymbol{x})\right) \mathbb{K}_{\delta}^{-1}(\boldsymbol{x})$, and $\mathbb{B}_{\delta}^{\mathrm{b}}(\boldsymbol{x})=\operatorname{det}\left(\mathbb{K}_{\delta}(\boldsymbol{x})\right)$. Lemma 2.2(ii) implies that (4.3) holds for these choices of $\mathbb{B}_{\delta}$. Let $f \in L^{1}(D ; \mathbb{R})$ and $\boldsymbol{g} \in L^{1}\left(D ; \mathbb{R}^{d}\right)$. We define the following families of mollification operators:

$$
\begin{aligned}
& \left(\mathcal{K}_{\delta, 0}^{\mathrm{g}} f\right)(\boldsymbol{x})=\int_{B(\mathbf{0}, 1)} \rho(\boldsymbol{y}) \widetilde{f}\left(\boldsymbol{\vartheta}_{\delta}(\boldsymbol{x})+(\delta \zeta) \boldsymbol{y}\right) \mathrm{d} y, \\
& \left(\mathcal{K}_{\delta, 0}^{\mathrm{c}} \boldsymbol{g}\right)(\boldsymbol{x})=\int_{B(\mathbf{0}, 1)} \rho(\boldsymbol{y}) \mathbb{K}_{\delta}^{\mathrm{T}}(\boldsymbol{x}) \widetilde{\boldsymbol{g}}\left(\boldsymbol{\vartheta}_{\delta}(\boldsymbol{x})+(\delta \zeta) \boldsymbol{y}\right) \mathrm{d} y, \\
& \left(\mathcal{K}_{\delta, 0}^{\mathrm{d}} \boldsymbol{g}\right)(\boldsymbol{x})=\int_{B(\mathbf{0}, 1)} \rho(\boldsymbol{y}) \operatorname{det}\left(\mathbb{K}_{\delta}(\boldsymbol{x})\right) \mathbb{K}_{\delta}^{-1}(\boldsymbol{x}) \widetilde{\boldsymbol{g}}\left(\boldsymbol{\vartheta}_{\delta}(\boldsymbol{x})+(\delta \zeta) \boldsymbol{y}\right) \mathrm{d} y, \\
& \left(\mathcal{K}_{\delta, 0}^{\mathrm{b}} f\right)(\boldsymbol{x})=\int_{B(\mathbf{0}, 1)} \rho(\boldsymbol{y}) \operatorname{det}\left(\mathbb{K}_{\delta}(\boldsymbol{x})\right) \widetilde{f}\left(\boldsymbol{\vartheta}_{\delta}(\boldsymbol{x})+(\delta \zeta) \boldsymbol{y}\right) \mathrm{d} y,
\end{aligned}
$$

for all $\boldsymbol{x} \in D$. Let $p \in[1, \infty]$. If $d=3$, we define

$$
\begin{aligned}
& \widetilde{Z}^{\mathrm{g}, p}(D)=\left\{f \in L^{p}(D) \mid \nabla \tilde{f} \in \boldsymbol{L}^{p}\left(\mathbb{R}^{d}\right)\right\}, \\
& \widetilde{\boldsymbol{Z}}^{\mathrm{c}, p}(D)=\left\{\boldsymbol{v} \in \boldsymbol{L}^{p}(D) \mid \nabla \times \widetilde{\boldsymbol{v}} \in \boldsymbol{L}^{p}\left(\mathbb{R}^{d}\right)\right\}, \\
& \widetilde{\boldsymbol{Z}}^{\mathrm{d}, p}(D)=\left\{\boldsymbol{v} \in \boldsymbol{L}^{p}(D) \mid \nabla \cdot \widetilde{\boldsymbol{v}} \in L^{p}\left(\mathbb{R}^{d}\right)\right\} .
\end{aligned}
$$

Lemma 4.2 (Commuting extension and derivatives). The following holds:

(i) $\nabla \widetilde{f}=\widetilde{\nabla f}$, for all $f \in \widetilde{Z}^{g, p}(D)$,

(ii) $\nabla \times \widetilde{\boldsymbol{g}}=\widetilde{\nabla \times \boldsymbol{g}}$, for all $\boldsymbol{g} \in \widetilde{\boldsymbol{Z}}^{\mathrm{c}, p}(D)$,

(iii) $\nabla \cdot \widetilde{\boldsymbol{g}}=\widetilde{\nabla \cdot \boldsymbol{g}}$, for all $\boldsymbol{g} \in \widetilde{\boldsymbol{Z}}^{\mathrm{d}, p}(D)$.

Proof. Let $f \in \widetilde{Z}^{\mathrm{g}, p}(D)$ and let $\boldsymbol{\psi} \in \boldsymbol{C}_{0}^{\infty}\left(\mathbb{R}^{d}\right)$ be a (vector-valued) smooth function compactly supported in $\operatorname{int}\left(\mathbb{R}^{d} \backslash D\right)$. Then,

$$
\int_{\mathbb{R}^{d}} \boldsymbol{\psi} \cdot \nabla \tilde{f} \mathrm{~d} x=-\int_{\mathbb{R}^{d}} \tilde{f} \nabla \cdot \boldsymbol{\psi} \mathrm{d} x=-\int_{D} f \nabla \cdot \boldsymbol{\psi} \mathrm{d} x=0 .
$$

Since $\boldsymbol{\psi}$ is arbitrary, this proves that $\nabla \tilde{f}$ is zero in $\mathbb{R}^{d} \backslash D$. Now let $\boldsymbol{\psi} \in C_{0}^{\infty}(D)$, then

$$
-\int_{D} \boldsymbol{\psi} \cdot \nabla \tilde{f} \mathrm{~d} x=-\int_{\mathbb{R}^{d}} \boldsymbol{\psi} \cdot \nabla \tilde{f} \mathrm{~d} x=\int_{\mathbb{R}^{d}} \tilde{f} \nabla \cdot \boldsymbol{\psi} \mathrm{d} x=\int_{D} f \nabla \cdot \boldsymbol{\psi} \mathrm{d} x=-\int_{D} \boldsymbol{\psi} \cdot \nabla f \mathrm{~d} x .
$$

Since $\psi$ is arbitrary, this proves that $(\nabla \widetilde{f})_{\mid D}=\nabla f$. We have thus proved that $\nabla \widetilde{f}=\widetilde{\nabla f}$. The argument for the other two equalities is identical.

Lemma 4.3 (Commuting with differential operators). The following holds:

(i) $\nabla \mathcal{K}_{\delta, 0}^{\mathrm{g}} f=\mathcal{K}_{\delta, 0}^{\mathrm{c}} \nabla f$, for all $f \in \widetilde{Z}^{\mathrm{g}, p}(D)$,

(ii) $\nabla \times \mathcal{K}_{\delta, 0}^{\mathrm{c}} \boldsymbol{g}=\mathcal{K}_{\delta, 0}^{\mathrm{d}} \nabla \times \boldsymbol{g}$, for all $\boldsymbol{g} \in \widetilde{\boldsymbol{Z}}^{\mathrm{c}, p}(D)$,

(iii) $\nabla \cdot \mathcal{K}_{\delta, 0}^{\mathrm{d}} \boldsymbol{g}=\mathcal{K}_{\delta, 0}^{\mathrm{b}} \nabla \cdot \boldsymbol{g}$, for all $\boldsymbol{g} \in \widetilde{\boldsymbol{Z}}^{\mathrm{d}, p}(D)$,

i.e., the following diagram commutes:

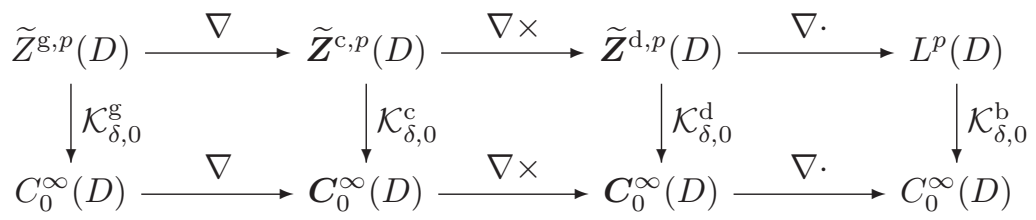


Proof. The proof is almost the same as that of Lemma 4.3. For instance, using the chain rule together with Lemma 4.2 , we obtain

$$
\begin{aligned}
\nabla \mathcal{K}_{\delta, 0}^{\mathrm{g}} f(\boldsymbol{x}) & =\int_{B(\mathbf{0}, 1)} \rho(\boldsymbol{y}) \mathbb{K}_{\delta}(\boldsymbol{x})^{\top} \nabla \tilde{f}(\boldsymbol{\vartheta}(\boldsymbol{x})+\delta \zeta \boldsymbol{y}) \mathrm{d} y \\
& =\int_{B(\mathbf{0}, 1)} \rho(\boldsymbol{y}) \mathbb{K}_{\delta}(\boldsymbol{x})^{\top} \widetilde{\nabla} f(\boldsymbol{\vartheta}(\boldsymbol{x})+\delta \zeta \boldsymbol{y}) \mathrm{d} y=\mathcal{K}_{\delta, 0}^{\mathrm{c}} \nabla f(\boldsymbol{x}) .
\end{aligned}
$$

Note here that it is critical that $\nabla \tilde{f}=\widetilde{\nabla f}$. The argument for the other two equalities is identical.

4.3. Convergence. Similarly to $§ 3.3$, we can now state convergence results. Owing to Lemma $2.2, \mathbb{K}_{\delta}$ and $\mathbb{K}_{\delta}^{-1}$ converge uniformly to the identity and $\operatorname{det}\left(\mathbb{K}_{\delta}\right)$ converges uniformly to 1 . As a result, there is $\tilde{\delta}_{0} \in(0,1]$ such that $\left\|\mathbb{K}_{\delta}-\mathbb{I}\right\|_{\ell^{2}} \leq \frac{1}{2}$, $\left\|\mathbb{K}_{\delta}^{-1}\right\|_{\ell^{2}} \leq 2$, and $\left|\operatorname{det}\left(\mathbb{K}_{\delta}^{-1}\right)\right| \leq 2^{d}$, for all $\delta \in\left[0, \tilde{\delta}_{0}\right]$ and all $\boldsymbol{x} \in D$. We combine the counterparts of Theorem 3.3 and Corollary 3.4 into one statement.

THEOREM 4.4 (Convergence). The sequence $\left(\mathcal{K}_{\delta, 0}\right)_{\delta \in\left[0, \tilde{\delta}_{0}\right]}$ is uniformly bounded in $\mathcal{L}\left(L^{p} ; L^{p}\right)$ for all $p \in[1, \infty]$. Moreover, for all $p \in[1, \infty)$

$$
\lim _{\delta \rightarrow 0}\left\|\mathcal{K}_{\delta, 0} f-f\right\|_{L^{p}\left(D ; \mathbb{R}^{q}\right)}=0, \quad \forall f \in L^{p}\left(D ; \mathbb{R}^{q}\right)
$$

and

$$
\begin{array}{cc}
\lim _{\delta \rightarrow 0}\left\|\nabla\left(\mathcal{K}_{\delta, 0}^{\mathrm{g}} f-f\right)\right\|_{\boldsymbol{L}^{p}(D)}=0, & \forall f \in \widetilde{Z}^{\mathrm{g}, p}(D), \\
\lim _{\delta \rightarrow 0}\left\|\nabla \times\left(\mathcal{K}_{\delta, 0}^{\mathrm{c}} \boldsymbol{g}-\boldsymbol{g}\right)\right\|_{\boldsymbol{L}^{p}(D)}=0, & \forall \boldsymbol{g} \in \widetilde{\boldsymbol{Z}}^{\mathrm{c}, p}(D), \\
\lim _{\delta \rightarrow 0}\left\|\nabla \cdot\left(\mathcal{K}_{\delta, 0}^{\mathrm{d}} \boldsymbol{g}-\boldsymbol{g}\right)\right\|_{L^{p}(D)}=0, & \forall \boldsymbol{g} \in \widetilde{\boldsymbol{Z}}^{\mathrm{d}, p}(D) .
\end{array}
$$

Proof. The proof of (4.7) is the same as that of Theorem 3.3. See the proof of Corollary 3.4 for the other three statements.

Let $s \in(0,1], p \in[1, \infty)$ or $s=1, p \in[1, \infty]$. Let us denote by $\widetilde{W^{s}, p}\left(D ; \mathbb{R}^{q}\right)$, the space composed of the functions in $W^{s, p}\left(D ; \mathbb{R}^{q}\right)$ whose extension by zero is in $W^{s, p}\left(\mathbb{R}^{d} ; \mathbb{R}^{q}\right)$. We set $|f|_{\widetilde{W}^{s, p}\left(D ; \mathbb{R}^{q}\right)}:=|\widetilde{f}|_{W^{s, p}\left(\mathbb{R}^{d} ; \mathbb{R}^{q}\right)}$.

TheOREM 4.5 (Convergence rate). There is $c$ such that

$$
\left\|\mathcal{K}_{\delta, 0} f-f\right\|_{L^{p}\left(D ; \mathbb{R}^{q}\right)} \leq c \delta^{s}|f|_{\widetilde{W}^{s, p}\left(D ; \mathbb{R}^{q}\right)},
$$

for all $f \in \widetilde{W}^{s, p}\left(D ; \mathbb{R}^{q}\right)$, all $\delta \in\left[0, \tilde{\delta}_{0}\right]$, and all $s \in(0,1], p \in[1, \infty)$ or $s=1$, $p \in[1, \infty]$.

Proof. The proof is identical to that of Theorem 3.5.

To state a convergence result using norms on $D$, we recall (see e.g., Grisvard [20, Thm. 1.4.2.4, Cor. 1.4.4.5]) that

$$
\begin{array}{ll}
\widetilde{W}^{s, p}\left(D ; \mathbb{R}^{q}\right)=W_{0}^{s, p}\left(D ; \mathbb{R}^{q}\right) & \text { if } s p \neq 1, \\
\widetilde{W}^{s, p}\left(D ; \mathbb{R}^{q}\right)=W^{s, p}\left(D ; \mathbb{R}^{q}\right) & \text { if } s p \in[0,1) .
\end{array}
$$

(Recall also that the constants in the above norm equivalences depend on $|s p-1|$.) 
Corollary 4.6 (Convergence rate on derivatives). Let $p \in[1, \infty)$ and $s \in\left(0, \frac{1}{p}\right)$. Then, there is $c$ (depending on $|s p-1|$ ) such that

$$
\begin{array}{rlrl}
\left\|\nabla\left(\mathcal{K}_{\delta, 0}^{\mathrm{g}} f-f\right)\right\|_{\boldsymbol{L}^{p}(D)} \leq c \delta^{s}\|\nabla f\|_{W^{s, p}(D)}, & & \forall f \in\left\{v \in L^{p}(D) \mid \nabla v \in \boldsymbol{W}^{s, p}(D)\right\}, \\
\left\|\nabla \times\left(\mathcal{K}_{\delta, 0}^{\mathrm{c}} \boldsymbol{g}-\boldsymbol{g}\right)\right\|_{\boldsymbol{L}^{p}(D)} \leq c \delta^{s}\|\nabla \times \boldsymbol{g}\|_{W^{s, p}(D)}, & \forall \boldsymbol{g} \in\left\{\boldsymbol{v} \in \boldsymbol{L}^{p}(D) \mid \nabla \times \boldsymbol{v} \in \boldsymbol{W}^{s, p}(D)\right\}, \\
\left\|\nabla \cdot\left(\mathcal{K}_{\delta, 0}^{\mathrm{d}} \boldsymbol{g}-\boldsymbol{g}\right)\right\|_{L^{p}(D)} \leq c \delta^{s}\|\nabla \cdot \boldsymbol{g}\|_{W^{s, p}(D)}, & \forall \boldsymbol{g} \in\left\{\boldsymbol{v} \in \boldsymbol{L}^{p}(D) \mid \nabla \cdot \boldsymbol{v} \in W^{s, p}(D)\right\} .
\end{array}
$$

Proof. The proof relies on the commuting properties from Lemma 4.3, Theorem 4.5 , and (4.10b). For instance,

$$
\begin{aligned}
\left\|\nabla\left(\mathcal{K}_{\delta, 0}^{\mathrm{g}} f-f\right)\right\|_{\boldsymbol{L}^{p}(D)} & =\left\|\mathcal{K}_{\delta, 0}^{\mathrm{c}} \nabla f-\nabla f\right\|_{\boldsymbol{L}^{p}(D)} & & \text { since } \nabla \mathcal{K}_{\delta, 0}^{\mathrm{g}}=\mathcal{K}_{\delta, 0}^{\mathrm{c}} \nabla \\
& \leq c \delta^{s}|\nabla f|_{\widetilde{W}^{s, p}(D)} & & \text { owing to Theorem 4.5 } \\
& \leq c_{s, p}^{\prime} \delta^{s}\|\nabla f\|_{\boldsymbol{W}^{s, p}(D)} & & \text { owing to (4.10b), }
\end{aligned}
$$

where $c_{s, p}^{\prime}$ depends on $|s p-1|$. Proceed similarly for the two other estimates. $\square$

Remark 4.1. The construction of $\mathcal{K}_{\delta, 0}^{\mathrm{c}}$ is similar in spirit to what has been proposed in Bonito et al. [7]. The curl estimates in Theorem 4.5 and Corollary 4.6 are identical to those in [7, Thm. 3.1].

Remark 4.2. $(s p>1)$ Convergence rates on derivatives can also be derived for $s p>1$, namely $\left\|\nabla\left(\mathcal{K}_{\delta, 0}^{\mathrm{g}} f-f\right)\right\|_{\boldsymbol{L}^{p}(D)} \leq c \delta^{s}|\nabla f|_{\boldsymbol{W}^{s, p}(D)}$ for all $f \in L^{p}(D)$ with $\nabla f \in \boldsymbol{W}_{0}^{s, p}(D),\left\|\nabla \times\left(\mathcal{K}_{\delta, 0}^{\mathrm{c}} \boldsymbol{g}-\boldsymbol{g}\right)\right\|_{\boldsymbol{L}^{p}(D)} \leq c \delta^{s}|\nabla \times \boldsymbol{g}|_{\boldsymbol{W}^{s, p}(D)}$ for all $\boldsymbol{g} \in \boldsymbol{L}^{p}(D)$ with $\nabla \times \boldsymbol{g} \in \boldsymbol{W}_{0}^{s, p}(D)$, and $\left\|\nabla \cdot\left(\mathcal{K}_{\delta, 0}^{\mathrm{d}} \boldsymbol{g}-\boldsymbol{g}\right)\right\|_{L^{p}(D)} \leq c \delta^{s}|\nabla \cdot \boldsymbol{g}|_{W^{s, p}(D)}$ for all $\boldsymbol{g} \in \boldsymbol{L}^{p}(D)$ with $\nabla \cdot \boldsymbol{g} \in W_{0}^{s, p}(D)$, where $c$ depends on $|s p-1|$. Note that these estimates require boundary conditions on the derivatives.

4.4. Traces of vector fields. In this section, we illustrate the use of the mollifying operator $\mathcal{K}_{\delta, 0}$. Let $p \in(1, \infty)$. Recall the spaces $\boldsymbol{Z}^{\mathrm{c}, p}(D)$ and $\boldsymbol{Z}^{\mathrm{d}, p}(D)$ from $(3.6 \mathrm{~b})$ (3.6c). Since the trace operator $\gamma_{0}: W^{1, p^{\prime}}(D) \longrightarrow W^{\frac{1}{p}, p^{\prime}}(\partial D)$ is surjective (see Brezis [8, p. 315], Grisvard [20, Thm. 1.5.1.2\&1.5.1.6], McLean [25, Thm. 3.38] (for $\left.\left.s \in\left(\frac{1}{2}, \frac{3}{2}\right), p=2\right)\right)$, letting $\langle\cdot, \cdot\rangle_{\partial D}$ denote the duality pairing between $\boldsymbol{W}^{-\frac{1}{p}, p}(\partial D)$ and $\boldsymbol{W}^{\frac{1}{p}, p^{\prime}}(\partial D)$, we define the bounded linear map $\gamma_{\times \boldsymbol{n}}: \boldsymbol{Z}^{\mathrm{c}, p}(D) \rightarrow \boldsymbol{W}^{-\frac{1}{p}, p}(\partial D)$ by

$$
\left\langle\gamma_{\times \boldsymbol{n}}(\boldsymbol{v}), \boldsymbol{l}\right\rangle_{\partial D}:=\int_{D} \boldsymbol{v} \cdot \nabla \times \boldsymbol{w}(\boldsymbol{l}) \mathrm{d} x-\int_{D} \boldsymbol{w}(\boldsymbol{l}) \cdot \nabla \times \boldsymbol{v} \mathrm{d} x
$$

for all $\boldsymbol{v} \in \boldsymbol{Z}^{\mathrm{c}, p}(D)$ and all $\boldsymbol{l} \in \boldsymbol{W}^{\frac{1}{p}, p^{\prime}}(\partial D)$, where $\boldsymbol{w}(\boldsymbol{l}) \in \boldsymbol{W}^{1, p^{\prime}}(D)$ is such that $\gamma_{0}(\boldsymbol{w}(\boldsymbol{l}))=\boldsymbol{l}$. Note that $\gamma_{\times \boldsymbol{n}}(\boldsymbol{v})=\boldsymbol{v}_{\mid \partial D} \times \boldsymbol{n}$ when $\boldsymbol{v}$ is smooth. The definition (4.11) is independent of the choice of $\boldsymbol{w}(\boldsymbol{l})$. Indeed, let $\boldsymbol{w}_{1}, \boldsymbol{w}_{2} \in \boldsymbol{W}^{1, p^{\prime}}(D)$ be such that $\gamma_{0}\left(\boldsymbol{w}_{1}\right)=\gamma_{0}\left(\boldsymbol{w}_{2}\right)=\boldsymbol{l}$, i.e., $\boldsymbol{w}_{1}-\boldsymbol{w}_{2} \in \boldsymbol{W}_{0}^{1, p^{\prime}}(D)$. Let $\left(\boldsymbol{\psi}_{n}\right)_{n \in \mathbb{N}}$ be a sequence in $\boldsymbol{C}_{0}^{\infty}(D)$ converging to $\boldsymbol{w}_{1}-\boldsymbol{w}_{2}$ in $\boldsymbol{W}_{0}^{1, p^{\prime}}(D)$. Then, $0=\int_{D} \boldsymbol{v} \cdot \nabla \times \boldsymbol{\psi}_{n} \mathrm{~d} x-\int_{D} \boldsymbol{\psi}_{n} \cdot \nabla \times \boldsymbol{v} \mathrm{d} x$, as can be seen by replacing $\boldsymbol{v}$ by $\mathcal{K}_{\delta}^{\mathrm{c}} \boldsymbol{v}$ and passing to the limit $\delta \rightarrow 0$. Passing to the limit $n \rightarrow \infty$ yields $0=\int_{D} \boldsymbol{v} \cdot \nabla \times\left(\boldsymbol{w}_{1}-\boldsymbol{w}_{2}\right) \mathrm{d} x-\int_{D}\left(\boldsymbol{w}_{1}-\boldsymbol{w}_{2}\right) \cdot \nabla \times \boldsymbol{v} \mathrm{d} x$; hence, $\left\langle\gamma_{\times \boldsymbol{n}}(\boldsymbol{v}), \gamma_{0}\left(\boldsymbol{w}_{1}\right)\right\rangle_{\partial D}=\left\langle\gamma_{\times \boldsymbol{n}}(\boldsymbol{v}), \gamma_{0}\left(\boldsymbol{w}_{2}\right)\right\rangle_{\partial D}$, which establishes the claim.

We also define $\gamma_{\cdot \boldsymbol{n}}: \boldsymbol{Z}^{\mathrm{d}, p}(D) \rightarrow W^{-\frac{1}{p}, p}(\partial D)$ by

$$
\left\langle\gamma_{\cdot \boldsymbol{n}}(\boldsymbol{v}), l\right\rangle_{\partial D}:=\int_{D} \boldsymbol{v} \cdot \nabla q(l) \mathrm{d} x+\int_{D} q(l) \nabla \cdot \boldsymbol{v} \mathrm{d} x
$$


for all $\boldsymbol{v} \in \boldsymbol{Z}^{\mathrm{d}, p}(D)$ and all $l \in W^{\frac{1}{p}, p^{\prime}}(\partial D)$, where $q(l) \in W^{1, p^{\prime}}(D)$ is such that $\gamma_{0}(q(l))=l$, and $\langle\cdot, \cdot\rangle_{\partial D}$ now denotes the duality pairing between $W^{-\frac{1}{p}, p}(\partial D)$ and $W^{\frac{1}{p}, p^{\prime}}(\partial D)$. Reasoning as above, one can verify that this definition is independent of the choice of $q(l)$. Note also that $\gamma_{\cdot \boldsymbol{n}}(\boldsymbol{v})=\boldsymbol{v}_{\mid \partial D} \cdot \boldsymbol{n}$ when $\boldsymbol{v}$ is smooth.

Let us now introduce

$$
\begin{aligned}
& \boldsymbol{Z}_{0}^{\mathrm{c}, p}(D):={\overline{\boldsymbol{C}_{0}^{\infty}(D)}}^{\boldsymbol{Z}^{\mathrm{c}, p}(D)}, \\
& \boldsymbol{Z}_{0}^{\mathrm{d}, p}(D):={\overline{\boldsymbol{C}_{0}^{\infty}(D)}}^{\mathrm{d}, p}(D)
\end{aligned}
$$

TheOREM 4.7 (Kernels of $\gamma_{\times \boldsymbol{n}}$ and $\left.\gamma_{\cdot \boldsymbol{n}}\right)$. Let $p \in(1, \infty)$. Then,

$$
\begin{aligned}
& \boldsymbol{Z}_{0}^{\mathrm{c}, p}(D)=\operatorname{ker}\left(\gamma_{\times \boldsymbol{n}}\right), \\
& \boldsymbol{Z}_{0}^{\mathrm{d}, p}(D)=\operatorname{ker}\left(\gamma_{\cdot \boldsymbol{n}}\right) .
\end{aligned}
$$

Proof. Let us do the proof for $\gamma_{\times \boldsymbol{n}}$, the proof for $\gamma_{\cdot \boldsymbol{n}}$ is similar.

(1) We first show that $\boldsymbol{Z}_{0}^{\mathrm{c}, p}(D) \subset \operatorname{ker}\left(\gamma_{\times \boldsymbol{n}}\right)$, which is the easiest to establish. By definition there is a sequence of smooth functions $\left(\boldsymbol{v}_{n}\right)_{n \in \mathbb{N}}$ in $\boldsymbol{C}_{0}^{\infty}(D)$ converging to $\boldsymbol{v}$ in $\boldsymbol{Z}^{\mathrm{c}, p}(D)$. Let $\boldsymbol{w}$ be a function in $\boldsymbol{C}^{\infty}(D) \cap \boldsymbol{C}^{0}(\bar{D})$, then

$$
0=\int_{D} \nabla \cdot\left(\boldsymbol{w} \times \boldsymbol{v}_{n}\right) \mathrm{d} x=\int_{D} \boldsymbol{v}_{n} \cdot \nabla \times \boldsymbol{w} \mathrm{d} x-\int_{D} \boldsymbol{w} \cdot \nabla \times \boldsymbol{v}_{n} \mathrm{~d} x .
$$

Both integrals on the right-hand side converge; hence,

$$
\left\langle\gamma_{\times \boldsymbol{n}}(\boldsymbol{v}), \gamma_{0}(\boldsymbol{w})\right\rangle_{\partial D}=\int_{D} \boldsymbol{v} \cdot \nabla \times \boldsymbol{w} \mathrm{d} x-\int_{D} \boldsymbol{w} \cdot \nabla \times \boldsymbol{v} \mathrm{d} x=0,
$$

for every function $\boldsymbol{w}$ in $\boldsymbol{C}^{\infty}(D) \cap \boldsymbol{C}^{0}(\bar{D})$. This also implies that the equality holds for all $\boldsymbol{w} \in W^{1, p^{\prime}}(D)$, since $\boldsymbol{C}^{\infty}(D) \cap \boldsymbol{C}^{0}(\bar{D})$ is dense in $\boldsymbol{w} \in W^{1, p^{\prime}}(D)$, see Lemma 3.1 and Theorem 3.3. In conclusion, $\boldsymbol{v} \in \operatorname{ker}\left(\gamma_{\times \boldsymbol{n}}\right)$ since $\gamma_{0}$ is surjective.

(2) Let us now establish the converse, i.e., $\operatorname{ker}\left(\gamma_{\times \boldsymbol{n}}\right) \subset \boldsymbol{Z}_{0}^{\mathrm{c}, p}(D)$. Let $\boldsymbol{v} \in \operatorname{ker}\left(\gamma_{\times \boldsymbol{n}}\right)$. Since $\boldsymbol{v} \in \boldsymbol{Z}^{\mathrm{c}, p}(D) \subset \boldsymbol{L}^{1}(D), \widetilde{\boldsymbol{v}}$ is differentiable in the distribution sense. Let $\boldsymbol{\psi} \in$ $C_{0}^{\infty}\left(\mathbb{R}^{d}\right)$, then

$$
\langle\nabla \times \widetilde{\boldsymbol{v}}, \boldsymbol{\psi}\rangle=\int_{\mathbb{R}^{d}} \widetilde{\boldsymbol{v}} \cdot \nabla \times \boldsymbol{\psi} \mathrm{d} x=\int_{D} \boldsymbol{v} \cdot \nabla \times \boldsymbol{\psi} \mathrm{d} x
$$

Using that $\boldsymbol{v} \in \operatorname{ker}\left(\gamma_{\times \boldsymbol{n}}\right)$, the above equality implies that

$$
\langle\nabla \times \widetilde{\boldsymbol{v}}, \boldsymbol{\psi}\rangle=\int_{D} \boldsymbol{v} \cdot \nabla \times \boldsymbol{\psi} \mathrm{d} x=\int_{D} \boldsymbol{\psi} \cdot \nabla \times \boldsymbol{v} \mathrm{d} x=\int_{\mathbb{R}^{d}} \boldsymbol{\psi} \cdot \widetilde{\nabla \times \boldsymbol{v}} \mathrm{d} x
$$

This proves that $\nabla \times \widetilde{\boldsymbol{v}}=\widetilde{\nabla \times \boldsymbol{v}} \in \boldsymbol{L}^{1}\left(\mathbb{R}^{d}\right)$. Hence $\boldsymbol{v} \in \widetilde{\boldsymbol{Z}}^{\mathrm{c}, p}(D)$. We can now apply (4.8b) from Theorem 4.4 since $\boldsymbol{v} \in \widetilde{\boldsymbol{Z}}^{\mathrm{c}, p}(D)$, i.e., the sequence $\left(\mathcal{K}_{\delta, 0}^{\mathrm{c}} \boldsymbol{v}\right)_{\delta \in\left[0, \tilde{\delta}_{0}\right]}$ converges to $\boldsymbol{v}$ in $\boldsymbol{Z}^{\mathrm{c}, p}(D)$. This proves that $\operatorname{ker}\left(\gamma_{\times \boldsymbol{n}}\right) \subset \boldsymbol{Z}_{0}^{\mathrm{c}, p}(D)$ since $\mathcal{K}_{\delta, 0}^{\mathrm{c}} \boldsymbol{v} \in \boldsymbol{C}_{0}^{\infty}(D)$ (see Lemma 4.1).

Remark 4.3. $\left(Z_{0}=\widetilde{Z}\right)$ The proof of Theorem 4.7 shows that $\boldsymbol{Z}_{0}^{\mathrm{c}, p}(D)=\widetilde{\boldsymbol{Z}}^{\mathrm{c}, p}(D)$ and $\boldsymbol{Z}_{0}^{\mathrm{d}, p}(D)=\widetilde{Z}^{\mathrm{d}, p}(D)$; similarly, $Z_{0}^{\mathrm{g}, p}(D)=\widetilde{Z}^{\mathrm{g}, p}(D)$.

5. Finite element setting. We introduce in this section the finite element setting that we are going to use in the rest of the paper. We henceforth assume that $D$ is a bounded polyhedron in $\mathbb{R}^{d}$. 
5.1. Meshes. Let $\left(\mathcal{T}_{h}\right)_{h>0}$ be a shape-regular sequence of affine meshes. To avoid technical questions regarding hanging nodes, we also assume that the meshes cover $D$ exactly and that they are matching, i.e., for all cells $K, K^{\prime} \in \mathcal{T}_{h}$ such that $K \neq K^{\prime}$ and $K \cap K^{\prime} \neq \emptyset$, the set $K \cap K^{\prime}$ is a common vertex, edge, or face of both $K$ and $K^{\prime}$ (with obvious extensions in higher space dimensions). Given a mesh $\mathcal{T}_{h}$, the elements in $K \in \mathcal{T}_{h}$ are closed sets in $\mathbb{R}^{d}$ by convention. The following sets

$$
\begin{aligned}
\mathcal{T}_{K} & :=\left\{K^{\prime} \in \mathcal{T}_{h} \mid K^{\prime} \cap K \neq \emptyset\right\}, \\
D_{K} & :=\operatorname{int}\left\{\boldsymbol{x} \in \bar{D} \mid \exists K^{\prime} \in \mathcal{T}_{K}, \boldsymbol{x} \in K^{\prime}\right\},
\end{aligned}
$$

for all $K \in \mathcal{T}_{h}$, will be invoked in the following sections. The set $\mathcal{T}_{K}$ is the union of all the cells that touch $K$, and $D_{K}$ is the interior of the collection of the points composing the cells in $\mathcal{T}_{K}$.

We assume that there is a reference element $\widehat{K}$ such that for any mesh $\mathcal{T}_{h}$ and any cell $K \in \mathcal{T}_{h}$, there is a bijective affine mapping $\boldsymbol{T}_{K}: \widehat{K} \longrightarrow K$ and an invertible matrix $\mathbb{J}_{K} \in \mathbb{R}^{d \times d}$ (not to be confused with $\mathbb{J}_{\delta}$ ) such that

$$
\boldsymbol{T}_{K}(\widehat{\boldsymbol{x}})-\boldsymbol{T}_{K}(\widehat{\boldsymbol{y}})=\mathbb{J}_{K}(\widehat{\boldsymbol{x}}-\widehat{\boldsymbol{y}}), \quad \forall \widehat{\boldsymbol{x}}, \widehat{\boldsymbol{y}} \in \widehat{K}
$$

The shape-regularity assumption of the mesh sequence implies that there are uniform constants $c^{\sharp}, c^{b}$ such that

$$
\left|\operatorname{det}\left(\mathbb{J}_{K}\right)\right|=|K||\widehat{K}|^{-1}, \quad\left\|\mathbb{J}_{K}\right\|_{\ell^{2}} \leq c^{\sharp} h_{K}, \quad\left\|\mathbb{J}_{K}^{-1}\right\|_{\ell^{2}} \leq c^{b} h_{K}^{-1},
$$

where $h_{K}$ is the diameter of $K$. It can be shown that $c^{\sharp}=\frac{1}{\rho_{\widehat{K}}}$ and $c^{b}=\frac{h_{K}}{\rho_{K}} h_{\widehat{K}}$ for meshes composed of simplices, where $\rho_{K}$ is the diameter of the largest ball that can be inscribed in $K, h_{\widehat{K}}$ is the diameter of $\widehat{K}$, and $\rho_{\widehat{K}}$ is the diameter of the largest ball that can be inscribed in $\widehat{K}$.

5.2. Definition of $\delta(\boldsymbol{x})$. In the arguments to follow, we are going to invoke smoothing operators like those defined in $\S 3$. To avoid having to assume that the mesh sequence is quasi-uniform, we construct a meshsize function $\mathfrak{h} \in C^{0,1}(\bar{D} ; \mathbb{R})$ such that there are three uniform constants $c, c^{\prime}, c^{\prime \prime}>0$ so that

$$
\|\mathfrak{h}\|_{W^{1, \infty}(D ; \mathbb{R})} \leq c, \quad c^{\prime} h_{K} \leq \mathfrak{h}(\boldsymbol{x}) \leq c^{\prime \prime} h_{K}, \quad \forall \boldsymbol{x} \in K,
$$

for all $K \in \mathcal{T}_{h}$. The construction of this function is standard in the finite element literature. For instance, if the mesh is composed of simplices, consider the piecewise linear function whose value at any vertex of the mesh is the average of the mesh-sizes of the simplices sharing this vertex.

Following Christiansen and Winther [12], we introduce $\epsilon \in(0,1)$ and define

$$
\delta(\boldsymbol{x}):=\epsilon \mathfrak{h}(\boldsymbol{x}), \quad \forall \boldsymbol{x} \in D
$$

Then we can define $\boldsymbol{\varphi}_{\delta}$ and $\boldsymbol{\vartheta}_{\delta}$ like in (2.1) and (2.3), and we can also define generic mollifying operators $\mathcal{K}_{\delta}$ and $\mathcal{K}_{\delta, 0}$ like in (3.3b) and (4.2). Lemmas $2.1 \& 2.2$ hold for $\ell \in\{0,1\}$ only, and the smoothness statement in Lemmas 3.1\&4.1 must be replaced by $\mathcal{K}_{\delta}(g) \in C^{1}\left(D ; \mathbb{R}^{q}\right)$ and $\mathcal{K}_{\delta, 0}(g) \in C_{0}^{1}\left(D ; \mathbb{R}^{q}\right)$ for all $g \in L^{1}\left(D ; \mathbb{R}^{q}\right)$, respectively, since $\delta$ is only Lipschitz. All the other statements in $\S 3$ and $\S 4$ remain unchanged. 
5.3. Reference and local finite elements. We are going to consider various approximation spaces based on the mesh sequence $\left(\mathcal{T}_{h}\right)_{h>0}$ and a fixed reference finite element $(\widehat{K}, \widehat{P}, \widehat{\Sigma})$. We henceforth assume that $\widehat{P}$ is composed of $\mathbb{R}^{q}$-valued functions for some integer $q \geq 1$ and that $\widehat{P} \subset W^{1, \infty}\left(\widehat{K} ; \mathbb{R}^{q}\right)$ (recall that $\widehat{P}$ is a space of polynomial functions in general). The reference degrees of freedom and the associated reference shape functions are denoted $\left\{\widehat{\sigma}_{1}, \ldots, \widehat{\sigma}_{n_{\mathrm{sh}}}\right\}$ and $\left\{\widehat{\theta}_{1}, \ldots, \widehat{\theta}_{n_{\mathrm{sh}}}\right\}$, respectively. We denote $\mathcal{N}:=\left\{1: n_{\mathrm{sh}}\right\}$ to alleviate the notation. We assume that the linear forms $\left\{\widehat{\sigma}_{i}\right\}_{i \in \mathcal{N}}$ can be extended to $\mathcal{L}(V(\widehat{K}) ; \mathbb{R})$, where $V(\widehat{K})$ is a Banach space such that $V(\widehat{K}) \subset L^{1}\left(\widehat{K} ; \mathbb{R}^{q}\right)$; see $\left[15\right.$, p. 39]. The interpolation operator $\mathcal{I}_{\widehat{K}}: V(\widehat{K}) \rightarrow \widehat{P}$ associated with the reference finite element $(\widehat{K}, \widehat{P}, \widehat{\Sigma})$ is defined by

$$
\mathcal{I}_{\widehat{K}}(\widehat{v})(\widehat{\boldsymbol{x}})=\sum_{i \in \mathcal{N}} \widehat{\sigma}_{i}(v) \widehat{\theta}_{i}(\widehat{\boldsymbol{x}}), \quad \forall \widehat{\boldsymbol{x}} \in \widehat{K}, \quad \forall \widehat{v} \in V(\widehat{K}) .
$$

By construction, $\mathcal{I}_{\widehat{K}} \in \mathcal{L}(V(\widehat{K}) ; \widehat{P})$, and $\widehat{P}$ is point-wise invariant by $\mathcal{I}_{\widehat{K}}$.

Let $K$ be a cell in the mesh $\mathcal{T}_{h}$. We introduce a $q \times q$ invertible matrix $\mathbb{A}_{K}$ and define the mapping $\psi_{K} \in \mathcal{L}\left(L^{1}\left(K ; \mathbb{R}^{q}\right) ; L^{1}\left(\widehat{K} ; \mathbb{R}^{q}\right)\right)$ by

$$
\psi_{K}(v)=\mathbb{A}_{K}\left(v \circ \boldsymbol{T}_{K}\right) .
$$

It can be shown (see [15, Prop. 1.61]) that upon setting

$$
\begin{aligned}
& P_{K}:=\left\{p=\psi_{K}^{-1}(\widehat{p}) \mid \widehat{p} \in \widehat{P}\right\}, \\
& \Sigma_{K}:=\left\{\sigma_{K, i}\right\}_{i \in \mathcal{N}} \text { s.t. } \sigma_{K, i}=\widehat{\sigma}_{i} \circ \psi_{K},
\end{aligned}
$$

the triple $\left(K, P_{K}, \Sigma_{K}\right)$ is a finite element. Moreover, the interpolation operator

$$
\mathcal{I}_{K}(v)(\boldsymbol{x})=\sum_{i \in \mathcal{N}} \sigma_{K, i}(v) \theta_{K, i}(\boldsymbol{x}), \quad \forall \boldsymbol{x} \in K, \quad \forall v \in V(K)
$$

where we have set $\theta_{K, i}:=\psi_{K}^{-1}\left(\widehat{\theta}_{i}\right)$, is such that $\mathcal{I}_{K} \in \mathcal{L}\left(V(K) ; P_{K}\right)$ and $P_{K}$ is pointwise invariant by $\mathcal{I}_{K}$. Definition (5.8a) implies that $P_{K} \subset W^{1, \infty}\left(K ; \mathbb{R}^{q}\right)$. More generally $\psi_{K}$ maps $W^{l, p}\left(K ; \mathbb{R}^{q}\right)$ to $W^{l, p}\left(\widehat{K} ; \mathbb{R}^{q}\right)$ for all $l \in \mathbb{N}$, all $p \in[1, \infty]$ (with $z^{ \pm \frac{1}{p}}=1, \forall z>0$ if $\left.p=\infty\right)$ and

$$
\begin{aligned}
\left|\psi_{K}\right|_{\mathcal{L}\left(W^{l, p}\left(K ; \mathbb{R}^{q}\right) ; W^{l, p}\left(\widehat{K} ; \mathbb{R}^{q}\right)\right)} & \leq c\left\|\mathbb{A}_{K}\right\|_{\ell^{2}}\left\|\mathbb{J}_{K}\right\|_{\ell^{2}}^{l}\left|\operatorname{det}\left(\mathbb{J}_{K}\right)\right|^{-\frac{1}{p}}, \\
\left|\psi_{K}^{-1}\right|_{\mathcal{L}\left(W^{l, p}\left(\widehat{K} ; \mathbb{R}^{q}\right) ; W^{l, p}\left(K ; \mathbb{R}^{q}\right)\right)} & \leq c\left\|\mathbb{A}_{K}^{-1}\right\|_{\ell^{2}}\left\|\mathbb{J}_{K}^{-1}\right\|_{\ell^{2}}^{l}\left|\operatorname{det}\left(\mathbb{J}_{K}\right)\right|^{\frac{1}{p}},
\end{aligned}
$$

for all $K \in \mathcal{T}_{h}$, (see e.g., [13, Thm. 3.1.2] or [15, Lemma 1.101]).

5.4. Structural assumptions. We henceforth assume that there is a uniform constant $c$ such that

$$
\left\|\mathbb{A}_{K}\right\|_{\ell^{2}}\left\|\mathbb{A}_{K}^{-1}\right\|_{\ell^{2}} \leq c\left\|\mathbb{J}_{K}\right\|_{\ell^{2}}\left\|\mathbb{J}_{K}^{-1}\right\|_{\ell^{2}}
$$

so that, owing to (5.3), $\left\|\mathbb{A}_{K}\right\|_{\ell^{2}}\left\|\mathbb{A}_{K}^{-1}\right\|_{\ell^{2}}$ is uniformly bounded with respect to $K$ and $h$. We also assume that the degrees of freedom over $\widehat{K}$ are either point values or integrals over edges, faces or $\widehat{K}$ itself. This is formalized by assuming that

$$
\left|\widehat{\sigma}_{i}(\widehat{v})\right| \leq c \begin{cases}\left\|\widehat{v}\left(\widehat{\boldsymbol{a}}_{i}\right)\right\|_{\ell^{2}\left(\mathbb{R}^{q}\right)} & \text { if point evaluation at } \widehat{\boldsymbol{a}}_{i}, \\ \frac{1}{\left|\widehat{S}_{\widehat{K}, i}\right|} \int_{\widehat{S}_{\widehat{K}, i}}\|\widehat{v}\|_{\ell^{2}\left(\mathbb{R}^{q}\right)} \mathrm{d} s, & \text { otherwise, }\end{cases}
$$


where $\widehat{S}_{\widehat{K}, i}$ is either an edge, a face, or $\widehat{K}$ itself. All these mesh-related geometric entities are assumed to be closed sets.

In the case of a point evaluation at $\widehat{\boldsymbol{a}}_{i}$, we observe that since the cardinal number of $\widehat{\Sigma}$ is finite, there exists a distance $\widehat{l}_{0}>0$ such that only one of the following situations occurs: (1) $\widehat{\boldsymbol{a}}_{i}$ is a vertex of $\widehat{K} ;(2) \widehat{\boldsymbol{a}}_{i}$ is in the interior of an edge of $\widehat{K}$ and is at least at distance $\widehat{l}_{0}$ from any vertex; (3) $\widehat{\boldsymbol{a}}_{i}$ is in the interior of a face of $\widehat{K}$ and is at least at distance $\widehat{l}_{0}$ from any edge; (4) $\widehat{\boldsymbol{a}}_{i}$ is in the interior of $\widehat{K}$ and is at least at distance $\widehat{l}_{0}$ from any face (with the obvious extension in higher space dimension).

Let $K \in \mathcal{T}_{h}$ and denote by $\left\{\boldsymbol{a}_{j}\right\}_{j \in \mathcal{M}_{K}}$ the collection of points associated with the degrees of freedom in $K$ defined by point evaluation. Note that there exists $\widehat{\boldsymbol{a}}_{i} \in \widehat{K}$ such that $\boldsymbol{a}_{j}=\boldsymbol{T}_{K}\left(\widehat{\boldsymbol{a}}_{i}\right)$ for all $j \in \mathcal{M}_{K}$. The shape-regularity of the mesh sequence implies that there is a constant $c_{\min }$ (uniform with respect to $j, K$, and $\mathcal{T}_{h}$ ) such that the open ball $B\left(\boldsymbol{a}_{j}, c_{\min } h_{K}\right)$ has the following property: for all $K^{\prime}$ such that $K^{\prime} \cap B\left(\boldsymbol{a}_{j}, c_{\min } h_{K}\right) \neq \emptyset$ and every $\boldsymbol{x} \in K^{\prime} \cap B\left(\boldsymbol{a}_{j}, c_{\min } h_{K}\right)$, the entire segment $\left[\boldsymbol{x}, \boldsymbol{a}_{j}\right]$ is in $K^{\prime}$. An immediate consequence of this observation is that

$$
\left\|v(\boldsymbol{x})-v\left(\boldsymbol{a}_{j}\right)\right\|_{\ell^{2}} \leq\left\|\boldsymbol{x}-\boldsymbol{a}_{j}\right\|_{\ell^{2}}\|\nabla v\|_{L^{\infty}\left(K^{\prime} ; \mathbb{R}^{q}\right)}, \quad \forall \boldsymbol{x} \in K^{\prime} \cap B\left(\boldsymbol{a}_{j}, c_{\min } h_{K}\right) \neq \emptyset,
$$

for all $v \in P_{K}$. Note that this implies that $B\left(\boldsymbol{a}_{j}, c_{\min } h_{K}\right) \subset \mathcal{T}_{K}$.

In the rest of the paper, we define $\epsilon_{\max }>0$ such that

$$
\begin{aligned}
& \max _{j \in \mathcal{M}_{K}} \max _{\boldsymbol{y} \in B(\mathbf{0}, 1)}\left\|\boldsymbol{a}_{j}-\left(\boldsymbol{\varphi}_{\delta\left(\boldsymbol{a}_{j}\right)}\left(\boldsymbol{a}_{j}\right)+r \delta\left(\boldsymbol{a}_{j}\right) \boldsymbol{y}\right)\right\|_{\ell^{2}} \leq c_{\min } h_{K}, \\
& \cup_{\boldsymbol{x} \in K}\left(\boldsymbol{\varphi}_{\delta(\boldsymbol{x})}(\boldsymbol{x})+r \delta(\boldsymbol{x}) B(\mathbf{0}, 1)\right) \subset D_{K},
\end{aligned}
$$

for all $K \in \mathcal{T}_{h}$, all $h>0$, and all functions $\delta$ satisfying (5.5) for any $\epsilon \in\left(0, \epsilon_{\max }\right]$.

5.5. Finite element spaces. We introduce the broken finite element space

$$
P^{\mathrm{b}}\left(\mathcal{T}_{h}\right)=\left\{v_{h} \in L^{1}\left(D ; \mathbb{R}^{q}\right) \mid \psi_{K}\left(v_{h \mid K}\right) \in \widehat{P}, \forall K \in \mathcal{T}_{h}\right\},
$$

where the statement $\psi_{K}\left(v_{h \mid K}\right) \in \widehat{P}$ is equivalent to $v_{h \mid K} \in P_{K}$. Notice also that $P^{\mathrm{b}}\left(\mathcal{T}_{h}\right) \subset W^{1, \infty}\left(\mathcal{T}_{h} ; \mathbb{R}^{q}\right):=\left\{v \in L^{\infty}\left(D ; \mathbb{R}^{q}\right) \mid v_{\mid K} \in W^{1, \infty}\left(K ; \mathbb{R}^{q}\right), \forall K \in \mathcal{T}_{h}\right\}$ since $P_{K} \subset W^{1, \infty}\left(K ; \mathbb{R}^{q}\right)$. We denote by $\mathcal{I}_{h}^{\mathrm{b}}: L^{p}(D) \rightarrow P^{\mathrm{b}}\left(\mathcal{T}_{h}\right)$ the interpolation operator such that $\mathcal{I}_{h}^{\mathrm{b}}(v)_{\mid K}=\mathcal{I}_{K}\left(v_{\mid K}\right)$, for all $K \in \mathcal{T}_{h}$.

We now introduce the notion of interfaces and jump across interfaces. We say that a subset $F \subset \bar{D}$ with a positive $(d-1)$-dimensional measure is an interface if there are distinct mesh cells $K_{l}, K_{r} \in \mathcal{T}_{h}$ such that $F=\partial K_{l} \cap \partial K_{r}$. We say that a subset $F \subset \bar{D}$ with positive $(d-1)$-dimensional measure is a boundary face if there is a mesh cell $K \in \mathcal{T}_{h}$ such that $F=\partial K \cap \partial D$. The unit normal vector $\boldsymbol{n}_{F}$ on $F$ is conventionally chosen to point from $K_{l}$ to $K_{r}$ for an interface and to point outward for a boundary face. The interfaces are collected in the set $\mathcal{F}_{h}^{\circ}$, the boundary faces are collected in the set $\mathcal{F}_{h}^{\partial}$, and we let $\mathcal{F}_{h}=\mathcal{F}_{h}^{\circ} \cup \mathcal{F}_{h}^{\partial}$. Let $F \in \mathcal{F}_{h}^{\circ}$ be a mesh interface, and let $K_{l}, K_{r}$ be the two cells such that $F=\partial K_{l} \cap \partial K_{r}$; the jump of $v \in W^{1,1}\left(\mathcal{T}_{h} ; \mathbb{R}^{q}\right)$ across $F$ is defined to be

$$
\llbracket v \rrbracket_{F}(\boldsymbol{x})=v_{\mid K_{l}}(\boldsymbol{x})-v_{\mid K_{r}}(\boldsymbol{x}) \quad \text { a.e. } \boldsymbol{x} \in F .
$$

Next we asume to have at hand a Banach space $W \subset L^{1}\left(D ; \mathbb{R}^{q}\right)$, with continuous embedding, where some notion of jump across interfaces makes sense. More precisely, we assume that there is a (bounded) linear trace operator $\gamma_{K}: W^{1,1}\left(K ; \mathbb{R}^{q}\right) \longrightarrow$ 
$L^{1}\left(\partial K ; \mathbb{R}^{t}\right)$, for some $t \geq 1$ and for all $K \in \mathcal{T}_{h}$, and we define the notion of $\gamma$-jump across interfaces as follows:

$$
\llbracket v \rrbracket_{F}^{\gamma}(\boldsymbol{x})=\gamma_{K_{l}}\left(v_{\mid K_{l}}\right)(\boldsymbol{x})-\gamma_{K_{r}}\left(v_{\mid K_{r}}\right)(\boldsymbol{x}) \quad \text { a.e. } \boldsymbol{x} \in F \text {. }
$$

We assume that $\left|\llbracket v \rrbracket_{F}^{\gamma}(\boldsymbol{x})\right| \leq\left|\llbracket v \rrbracket_{F}(\boldsymbol{x})\right|$, for a.e. $\boldsymbol{x} \in F$, for all $v \in W^{1,1}\left(\mathcal{T}_{h}\right)$, so that

$$
v \in W^{1,1}\left(D ; \mathbb{R}^{q}\right) \Longrightarrow\left(\llbracket v \rrbracket_{F}^{\gamma}=0, \forall F \in \mathcal{F}_{h}^{\circ}\right) .
$$

We relate the notion of $\gamma$-jump to the space $W$ by assuming that

$$
v \in W \cap W^{1,1}\left(\mathcal{T}_{h} ; \mathbb{R}^{q}\right) \Longrightarrow\left(\llbracket v \rrbracket_{F}^{\gamma}=0, \forall F \in \mathcal{F}_{h}^{\circ}\right),
$$

and, conversely, that a function in $W^{1, \infty}\left(\mathcal{T}_{h} ; \mathbb{R}^{q}\right)$ with zero $\gamma$-jumps across interfaces is in $W$. With this setting, we define

$$
P\left(\mathcal{T}_{h}\right):=P^{\mathrm{b}}\left(\mathcal{T}_{h}\right) \cap W .
$$

The above assumptions imply that

$$
P\left(\mathcal{T}_{h}\right)=\left\{v_{h} \in P^{\mathrm{b}}\left(\mathcal{T}_{h}\right) \mid \llbracket v_{h} \rrbracket_{F}^{\gamma}=0, \forall F \in \mathcal{F}_{h}^{\circ}\right\} .
$$

Let $F \in \mathcal{F}_{h}^{\partial}$ be a boundary face and denote by $K_{F}$ the unique cell such that $F \subset \partial K_{F}$. We consider the global trace operator $\gamma: W^{1,1}\left(D ; \mathbb{R}^{q}\right) \longrightarrow L^{1}\left(\partial D ; \mathbb{R}^{t}\right)$ such that

$$
\gamma(v)_{\mid F}=\gamma_{K_{F}}\left(v_{\mid K_{F}}\right), \quad \forall F \in \mathcal{F}_{h}^{\partial} .
$$

We assume that $\gamma$ can be extended to $W$ into a bounded linear operator $\gamma: W \longrightarrow W^{\partial}$ where $W^{\partial}$ is an appropriate Banach space, whose exact structure is not important for the time being. We define $W_{0}=\operatorname{ker}(\gamma)$, i.e., $W_{0}=\{v \in W \mid \gamma(v)=0\}$. Let us introduce $P_{0}\left(\mathcal{T}_{h}\right)=P\left(\mathcal{T}_{h}\right) \cap W_{0}$ :

$$
P_{0}\left(\mathcal{T}_{h}\right):=\left\{v_{h} \in P\left(\mathcal{T}_{h}\right) \mid \gamma\left(v_{h}\right)=0\right\} .
$$

5.6. Examples. The present theory covers a large class of scalar- and vectorvalued finite elements like Lagrange, Nédélec, and Raviart-Thomas finite elements. To remain general, we denote the three reference elements corresponding to the above three classes as follows: $\left(\widehat{K}, \widehat{P}^{\mathrm{g}}, \widehat{\Sigma}^{\mathrm{g}}\right),\left(\widehat{K}, \widehat{\boldsymbol{P}}^{\mathrm{c}}, \widehat{\Sigma}^{\mathrm{c}}\right)$ and $\left(\widehat{K}, \widehat{\boldsymbol{P}}^{\mathrm{d}}, \widehat{\Sigma}^{\mathrm{d}}\right)$. The corresponding domains for the degrees of freedom are denoted $V^{\mathrm{g}}(\widehat{K}), \boldsymbol{V}^{\mathrm{c}}(\widehat{K}), \boldsymbol{V}^{\mathrm{d}}(\widehat{K})$. We think of $\left(\widehat{K}, \widehat{P}^{\mathrm{g}}, \widehat{\Sigma}^{\mathrm{g}}\right)$ as a scalar-valued finite element $(q=1)$ and some of its degrees of freedom require point evaluation, for instance $\left(\widehat{K}, \widehat{P}^{\mathrm{g}}, \widehat{\Sigma}^{\mathrm{g}}\right)$ could be a Lagrange element. We assume that the finite element $\left(\widehat{K}, \widehat{\boldsymbol{P}}^{\mathrm{c}}, \widehat{\Sigma}^{\mathrm{c}}\right)$ is vector-valued $(q=d)$ and some of its degrees of freedom require to evaluate integrals over edges. Typically, $\left(\widehat{K}, \widehat{\boldsymbol{P}}^{\mathrm{c}}, \widehat{\Sigma}^{\mathrm{c}}\right)$ is a Nédélec-type or edge element. Likewise, the finite element $\left(\widehat{K}, \widehat{\boldsymbol{P}}^{\mathrm{d}}, \widehat{\Sigma}^{\mathrm{d}}\right)$ is assumed to be vector-valued $(q=d)$ and some of its degrees of freedom are assumed to require evaluation of integrals over faces. Typically, $\left(\widehat{K}, \widehat{\boldsymbol{P}}^{\mathrm{d}}, \widehat{\Sigma}^{\mathrm{d}}\right)$ is a Raviart-Thomas-type element. The arguments developed herein do not require to know the exact structure of the above elements.

The above assumptions imply that it is admissible to choose $V^{\mathrm{g}}(\widehat{K})=W^{s, p}(\widehat{K})$ with $s>\frac{d}{p}, \boldsymbol{V}^{\mathrm{c}}(\widehat{K})=\boldsymbol{W}^{s, p}(\widehat{K})$ with $s>\frac{d-1}{p}$, and $\boldsymbol{V}^{\mathrm{d}}(\widehat{K})=\boldsymbol{W}^{s, p}(\widehat{K})$ with $s>\frac{1}{p}$ 
(recall that denoting by $M$ a smooth manifold of dimension $d^{\prime}$ in $\widehat{K}$, the restriction operator to $M$ is continuous from $W^{s, p}(\widehat{K})$ to $L^{p}(M)$ provided $\left.s>\frac{d-d^{\prime}}{p}\right)$. Note that it is also legitimate to choose

$$
V^{\mathrm{g}}(\widehat{K})=W^{d, 1}(\widehat{K}), \quad \boldsymbol{V}^{\mathrm{d}}(\widehat{K})=\boldsymbol{W}^{1,1}(\widehat{K}), \quad \boldsymbol{V}^{\mathrm{c}}(\widehat{K})=\boldsymbol{W}^{d-1,1}(\widehat{K}),
$$

since $\left.W^{d, 1}(\widehat{K}) \subset C^{0}(\widehat{K})\right)$, functions in $W^{1,1}(\widehat{K})$ have a trace in $L^{1}(\partial \widehat{K})$, and functions in $W^{d-1,1}(\widehat{K})$ have integrable traces on the one-dimensional edges of $\widehat{K}$.

Let $\psi_{K}^{\mathrm{g}}, \boldsymbol{\psi}_{K}^{\mathrm{c}}, \boldsymbol{\psi}_{K}^{\mathrm{d}}$ be the linear maps introduced in (5.7) for each of the reference finite elements defined above. In practice $\psi_{K}^{\mathrm{g}}$ is the pullback by $\boldsymbol{T}_{K}$, and $\boldsymbol{\psi}_{K}^{\mathrm{c}}$ and $\boldsymbol{\psi}_{K}^{\mathrm{d}}$ are the contravariant and covariant Piola transformations, respectively, i.e.,

$$
\begin{array}{ll}
\mathbb{A}_{K}^{\mathrm{g}}=1, & \psi_{K}^{\mathrm{g}}(v)=v \circ \boldsymbol{T}_{K}, \\
\mathbb{A}_{K}^{\mathrm{c}}=\mathbb{J}_{K}^{\mathrm{T}}, & \boldsymbol{\psi}_{K}^{\mathrm{c}}(\boldsymbol{v})=\mathbb{J}_{K}^{\mathrm{T}}\left(\boldsymbol{v} \circ \boldsymbol{T}_{K}\right), \\
\mathbb{A}_{K}^{\mathrm{d}}=\operatorname{det}\left(\mathbb{J}_{K}\right) \mathbb{J}_{K}^{-1}, & \boldsymbol{\psi}_{K}^{\mathrm{d}}(\boldsymbol{v})=\operatorname{det}\left(\mathbb{J}_{K}\right) \mathbb{J}_{K}^{-1}\left(\boldsymbol{v} \circ \boldsymbol{T}_{K}\right) .
\end{array}
$$

Note that $c=1$ in (5.11) for the above examples. We consider the following $\gamma$-traces:

$$
\begin{aligned}
\gamma_{K}^{\mathrm{g}}\left(v_{\mid K}\right)(\boldsymbol{x}):=v_{\mid K}(\boldsymbol{x}), & & \forall \boldsymbol{x} \in F, \\
\gamma_{K}^{\mathrm{c}}\left(\boldsymbol{v}_{\mid K}\right)(\boldsymbol{x}):=\boldsymbol{v}_{\mid K}(\boldsymbol{x}) \times \boldsymbol{n}_{F}, & & \forall \boldsymbol{x} \in F, \\
\gamma_{K}^{\mathrm{d}}\left(\boldsymbol{v}_{\mid K}\right)(\boldsymbol{x}):=\boldsymbol{v}_{\mid K}(\boldsymbol{x}) \cdot \boldsymbol{n}_{F}, & & \forall \boldsymbol{x} \in F,
\end{aligned}
$$

and the following finite element spaces:

$$
\begin{aligned}
& P^{\mathrm{g}}\left(\mathcal{T}_{h}\right):=\left\{v_{h} \in L^{1}(D) \mid \psi_{K}^{\mathrm{g}}\left(v_{h \mid K}\right) \in \widehat{P}^{\mathrm{g}}, \forall K \in \mathcal{T}_{h}, \llbracket v_{h} \rrbracket_{F}^{\mathrm{g}}=0, \forall F \in \mathcal{F}_{h}^{\circ}\right\}, \\
& \boldsymbol{P}^{\mathrm{c}}\left(\mathcal{T}_{h}\right):=\left\{\boldsymbol{v}_{h} \in \boldsymbol{L}^{1}(D) \mid \boldsymbol{\psi}_{K}^{\mathrm{c}}\left(\boldsymbol{v}_{h \mid K}\right) \in \widehat{\boldsymbol{P}}^{\mathrm{c}}, \forall K \in \mathcal{T}_{h}, \llbracket \boldsymbol{v}_{h} \rrbracket_{F}^{\mathrm{c}}=\mathbf{0}, \forall F \in \mathcal{F}_{h}^{\circ}\right\}, \\
& \boldsymbol{P}^{\mathrm{d}}\left(\mathcal{T}_{h}\right):=\left\{\boldsymbol{v}_{h} \in \boldsymbol{L}^{1}(D) \mid \boldsymbol{\psi}_{K}^{\mathrm{d}}\left(\boldsymbol{v}_{h \mid K}\right) \in \widehat{\boldsymbol{P}}^{\mathrm{d}}, \forall K \in \mathcal{T}_{h}, \llbracket \boldsymbol{v}_{h} \rrbracket_{F}^{\mathrm{d}}=0, \forall F \in \mathcal{F}_{h}^{\circ}\right\},
\end{aligned}
$$

where we simplified the notation by using $\llbracket v_{h} \rrbracket_{F}^{\mathrm{g}}$ instead of $\llbracket v_{h} \rrbracket_{F}^{\gamma^{\mathrm{g}}}$, etc. Note the conformity properties $P^{\mathrm{g}}\left(\mathcal{T}_{h}\right) \subset Z^{\mathrm{g}, p}(D), \boldsymbol{P}^{\mathrm{c}}\left(\mathcal{T}_{h}\right) \subset \boldsymbol{Z}^{\mathrm{c}, p}(D)$, and $\boldsymbol{P}^{\mathrm{d}}\left(\mathcal{T}_{h}\right) \subset \boldsymbol{Z}^{\mathrm{d}, p}(D)$. Likewise, observing that $Z_{0}^{\mathrm{g}, p}(D):=\left\{v \in Z^{\mathrm{g}, p}(D) \mid \gamma^{\mathrm{g}}(v)=0\right\}$ etc., we define

$$
\begin{aligned}
& P_{0}^{\mathrm{g}}\left(\mathcal{T}_{h}\right):=P^{\mathrm{g}}\left(\mathcal{T}_{h}\right) \cap Z_{0}^{\mathrm{g}, p}(D), \\
& \boldsymbol{P}_{0}^{\mathrm{c}}\left(\mathcal{T}_{h}\right):=\boldsymbol{P}^{\mathrm{c}}\left(\mathcal{T}_{h}\right) \cap \boldsymbol{Z}_{0}^{\mathrm{c}, p}(D), \\
& \boldsymbol{P}_{0}^{\mathrm{d}}\left(\mathcal{T}_{h}\right):=\boldsymbol{P}^{\mathrm{d}}\left(\mathcal{T}_{h}\right) \cap \boldsymbol{Z}_{0}^{\mathrm{d}, p}(D) .
\end{aligned}
$$

We finally denote by $\mathcal{I}_{h}^{\mathrm{g}}, \mathcal{I}_{h}^{\mathrm{d}}, \mathcal{I}_{h}^{\mathrm{c}}$ and $\mathcal{I}_{h 0}^{\mathrm{g}}, \mathcal{I}_{h 0}^{\mathrm{d}}, \mathcal{I}_{h 0}^{\mathrm{c}}$ the canonical interpolation operators associated with the finite element spaces $P^{\mathrm{g}}\left(\mathcal{T}_{h}\right), \boldsymbol{P}^{\mathrm{c}}\left(\mathcal{T}_{h}\right), \boldsymbol{P}^{\mathrm{d}}\left(\mathcal{T}_{h}\right)$, and $P_{0}^{\mathrm{g}}\left(\mathcal{T}_{h}\right), \boldsymbol{P}_{0}^{\mathrm{c}}\left(\mathcal{T}_{h}\right), \boldsymbol{P}_{0}^{\mathrm{d}}\left(\mathcal{T}_{h}\right)$. Note that $\mathcal{I}_{h}^{\mathrm{g}}: W^{s, p}(D) \longrightarrow P^{\mathrm{g}}\left(\mathcal{T}_{h}\right) \subset L^{p}(D)$ is stable provided $s>\frac{d}{p}, \mathcal{I}_{h}^{\mathrm{c}}: \boldsymbol{W}^{s, p}(D) \longrightarrow \boldsymbol{P}^{\mathrm{c}}\left(\mathcal{T}_{h}\right) \subset \boldsymbol{L}^{p}(D)$ is stable provided $s>\frac{d-1}{p}$ $\left(=\frac{2}{p}\right.$ for $\left.d=3\right)$, and $\mathcal{I}_{h}^{\mathrm{d}}: \boldsymbol{W}^{s, p}(D) \longrightarrow \boldsymbol{P}^{\mathrm{d}}\left(\mathcal{T}_{h}\right) \subset \boldsymbol{L}^{p}(D)$ is stable provided $s>$ $\frac{d-(d-1)}{p}=\frac{1}{p}$. We finally assume that the polynomial degrees in each of these spaces are compatible so that the following commuting properties hold with $s>\frac{d}{p}$ :

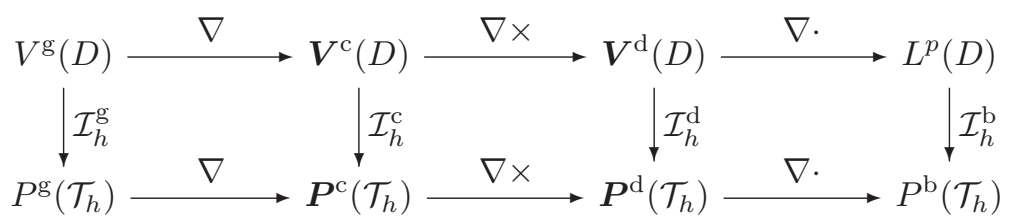


where

$$
\begin{aligned}
V^{\mathrm{g}}(D) & =\left\{f \in W^{s, p}(D) \mid \nabla f \in \boldsymbol{W}^{s-\frac{1}{p}, p}(D)\right\}, \\
\boldsymbol{V}^{\mathrm{c}}(D) & =\left\{\boldsymbol{g} \in \boldsymbol{W}^{s-\frac{1}{p}, p}(D) \mid \nabla \times \boldsymbol{g} \in \boldsymbol{W}^{s-\frac{2}{p}, p}(D)\right\}, \\
\boldsymbol{V}^{\mathrm{d}}(D) & =\left\{\boldsymbol{g} \in \boldsymbol{W}^{s-\frac{2}{p}, p}(D) \mid \nabla \cdot \boldsymbol{g} \in W^{s-\frac{3}{p}, p}(D)\right\},
\end{aligned}
$$

and $\mathcal{I}_{h}^{\mathrm{b}}$ is an interpolation operator only involving integrals over mesh cells. Likewise, upon introducing

$$
\begin{aligned}
V_{0}^{\mathrm{g}}(D) & =\left\{f \in V^{\mathrm{g}}(D) \mid f_{\mid \partial D}=0\right\}, \\
\boldsymbol{V}_{0}^{\mathrm{c}}(D) & =\left\{\boldsymbol{g} \in \boldsymbol{V}^{\mathrm{c}}(D) \mid \boldsymbol{g} \times \boldsymbol{n}_{\mid \partial D}=\mathbf{0}\right\}, \\
\boldsymbol{V}_{0}^{\mathrm{d}}(D) & =\left\{\boldsymbol{g} \in \boldsymbol{V}^{\mathrm{d}}(D) \mid \boldsymbol{g} \cdot \boldsymbol{n}_{\mid \partial D}=0\right\},
\end{aligned}
$$

we assume that the following diagram commutes:

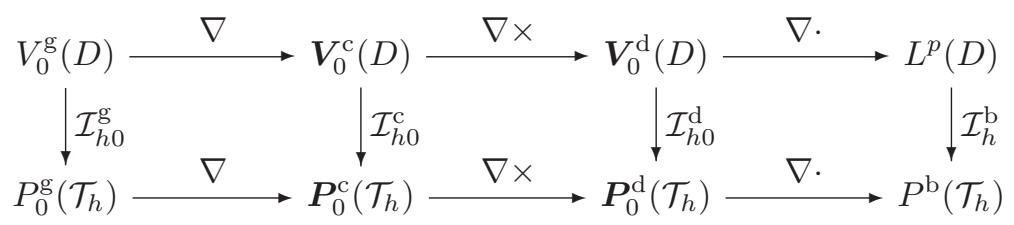

6. Stable, commuting, quasi-interpolation projection. We introduce in this section a family of finite-element-based quasi-interpolation operators (with and without boundary conditions) that are $L^{p}$-stable, commute with the standard differential operators $\nabla, \nabla \times$, and $\nabla \cdot$, and preserve the above finite element spaces.

6.1. The operator $\mathcal{I}_{h} \mathcal{K}_{\delta}$. Owing to the properties of the smoothing operators established above, it makes sense to consider the discrete functions $\mathcal{I}_{h}^{\mathrm{g}} \mathcal{K}_{\delta}^{\mathrm{g}} f \in P^{\mathrm{g}}\left(\mathcal{T}_{h}\right)$, $\mathcal{I}_{h 0}^{\mathrm{g}} \mathcal{K}_{\delta}^{\mathrm{g}} f \in P_{0}^{\mathrm{g}}\left(\mathcal{T}_{h}\right), \mathcal{I}_{h}^{\mathrm{b}} \mathcal{K}_{\delta}^{\mathrm{b}} f \in P^{\mathrm{b}}\left(\mathcal{T}_{h}\right), \mathcal{I}_{h}^{\mathrm{c}} \mathcal{K}_{\delta}^{\mathrm{c}} \boldsymbol{g} \in \boldsymbol{P}^{\mathrm{c}}\left(\mathcal{T}_{h}\right), \mathcal{I}_{h 0}^{\mathrm{c}} \mathcal{K}_{\delta}^{\mathrm{c}} \boldsymbol{g} \in \boldsymbol{P}_{0}^{\mathrm{c}}\left(\mathcal{T}_{h}\right), \mathcal{I}_{h}^{\mathrm{d}} \mathcal{K}_{\delta}^{d} \boldsymbol{g} \in$ $\boldsymbol{P}^{\mathrm{d}}\left(\mathcal{T}_{h}\right)$ and $\mathcal{I}_{h 0}^{\mathrm{d}} \mathcal{K}_{\delta}^{d} \boldsymbol{g} \in \boldsymbol{P}_{0}^{\mathrm{d}}\left(\mathcal{T}_{h}\right)$ for any integrable scalar-valued function $f$ and any integrable vector-valued function $\boldsymbol{g}$. We now establish some stability properties of the restrictions of the operators $\mathcal{I}_{h}^{\mathrm{g}} \mathcal{K}_{\delta}^{\mathrm{g}}, \mathcal{I}_{h 0}^{\mathrm{g}} \mathcal{K}_{\delta}^{\mathrm{g}}, \mathcal{I}_{h}^{\mathrm{b}} \mathcal{K}_{\delta}^{\mathrm{b}}, \mathcal{I}_{h}^{\mathrm{c}} \mathcal{K}_{\delta}^{\mathrm{c}}, \mathcal{I}_{h 0}^{\mathrm{c}} \mathcal{K}_{\delta}^{\mathrm{c}}, \mathcal{I}_{h}^{\mathrm{d}} \mathcal{K}_{\delta}^{d}$, and $\mathcal{I}_{h 0}^{\mathrm{d}} \mathcal{K}_{\delta}^{d}$ to the discrete spaces $P^{\mathrm{g}}\left(\mathcal{T}_{h}\right), P_{0}^{\mathrm{g}}\left(\mathcal{T}_{h}\right), P^{\mathrm{b}}\left(\mathcal{T}_{h}\right), \boldsymbol{P}^{\mathrm{c}}\left(\mathcal{T}_{h}\right), \boldsymbol{P}_{0}^{\mathrm{c}}\left(\mathcal{T}_{h}\right), \boldsymbol{P}^{\mathrm{d}}\left(\mathcal{T}_{h}\right)$, and $\boldsymbol{P}_{0}^{\mathrm{d}}\left(\mathcal{T}_{h}\right)$.

To avoid repeating proofs seven times, we denote by $\mathcal{I}_{h}$ one of the interpolation operators introduced above and $\mathcal{K}_{\delta}$ the corresponding smoothing operator; likewise, the range of $\mathcal{I}_{h}$ is denoted $P\left(\mathcal{T}_{h}\right)$. We assume that $P\left(\mathcal{T}_{h}\right)$ is composed of $\mathbb{R}^{q}$-valued fields.

Remark 6.1. (Boundary conditions) Note that we do not invoke $\mathcal{K}_{\delta, 0}^{\mathrm{g}}, \mathcal{K}_{\delta, 0}^{\mathrm{c}}$, and $\mathcal{K}_{\delta, 0}^{\mathrm{d}}$ in the above construction. The theory to be exposed in the next section holds by using $\mathcal{K}_{\delta}^{\mathrm{g}}, \mathcal{K}_{\delta}^{\mathrm{c}}$, and $\mathcal{K}_{\delta}^{\mathrm{d}}$ in all the cases, whether homogeneous boundary conditions are enforced or not in the discrete spaces.

6.2. $L^{p}$-stability of the operator $\mathcal{I}_{h} \mathcal{K}_{\delta}$. We start with a key result in the spirit of [12, Lem. 4.2], see also [29, Lem. 6]. This result is crucial to devise a quasiinterpolation operator that preserves the finite element space $P\left(\mathcal{T}_{h}\right)$.

LEMma 6.1 (Discrete $L^{p}$-approximation). There is $c_{\text {stab }}>0$, uniform with respect to the mesh sequence, such that $\left\|f_{h}-\mathcal{I}_{h} \mathcal{K}_{\delta} f_{h}\right\|_{L^{p}\left(D ; \mathbb{R}^{q}\right)} \leq c_{\mathrm{stab}} \epsilon\left\|f_{h}\right\|_{L^{p}\left(D ; \mathbb{R}^{q}\right)}$ for all $\epsilon \in\left(0, \epsilon_{\max }\right]$, all $f_{h} \in P\left(\mathcal{T}_{h}\right)$ and all $p \in[1, \infty]$.

Proof. (1) Let $f_{h} \in P\left(\mathcal{T}_{h}\right)$ and let us set $e_{h}:=f_{h}-\mathcal{I}_{h} \mathcal{K}_{\delta} f_{h}$ and $e:=f_{h}-\mathcal{K}_{\delta} f_{h}$; note that $e_{h}=\mathcal{I}_{h} e$. Let $K$ be a cell in $\mathcal{T}_{h}$, then using that $\theta_{K, i}:=\psi_{K}^{-1}\left(\widehat{\theta}_{i}\right)$, we have 
$\left\|\theta_{K, i}\right\|_{L^{p}\left(K ; \mathbb{R}^{q}\right)} \leq \operatorname{det}\left(\mathbb{J}_{K}\right)^{\frac{1}{p}}\left\|\mathbb{A}_{K}^{-1}\right\|_{\ell^{2}}\left\|\widehat{\theta}_{i}\right\|_{L^{p}\left(\widehat{K} ; \mathbb{R}^{q}\right)}$ for all $i \in\left\{1: n_{\mathrm{sh}}\right\}$, and we infer that

$$
\begin{aligned}
\left\|e_{h}\right\|_{L^{p}\left(K ; \mathbb{R}^{q}\right)}=\left\|\mathcal{I}_{h} e\right\|_{L^{p}\left(K ; \mathbb{R}^{q}\right)} & \leq \sum_{i \in \mathcal{N}}\left|\sigma_{K, i}(e)\right|\left\|\theta_{K, i}\right\|_{L^{p}\left(K ; \mathbb{R}^{q}\right)} \\
& \leq \operatorname{det}\left(\mathbb{J}_{K}\right)^{\frac{1}{p}}\left\|\mathbb{A}_{K}^{-1}\right\|_{\ell^{2}} \sum_{i \in \mathcal{N}}\left|\sigma_{K, i}(e)\right|,
\end{aligned}
$$

The rest of the proof consists of estimating $\sigma_{K, i}(e)$.

(2) Let us assume first that the degree of freedom $\sigma_{K, i}$ is a value at a point $\boldsymbol{a}_{j}:=\boldsymbol{T}_{K}\left(\widehat{\boldsymbol{a}}_{i}\right)$ in $K$. Then using the assumption (5.12) and the definition (5.7) of $\psi_{K}$, we infer that $\left|\sigma_{K, i}(e)\right| \leq c\left\|\mathbb{A}_{K}\right\|_{\ell^{2}}\left\|e\left(\boldsymbol{a}_{j}\right)\right\|_{\ell^{2}}$. By proceeding as in the proof of Theorem 3.3 (step (2)), we obtain

$$
e\left(\boldsymbol{a}_{j}\right)=f_{h}\left(\boldsymbol{a}_{j}\right)-\mathcal{K}_{\delta} f_{h}\left(\boldsymbol{a}_{j}\right)=\int_{B(\mathbf{0}, 1)} \rho(\boldsymbol{y})\left(f_{h}\left(\boldsymbol{a}_{j}\right)-f_{h}\left(\boldsymbol{\varphi}_{\delta\left(\boldsymbol{a}_{j}\right)}\left(\boldsymbol{a}_{j}\right)+\delta\left(\boldsymbol{a}_{j}\right) r \boldsymbol{y}\right)\right) \mathrm{d} y .
$$

Owing to (5.13) and (5.14a) (recall that $\epsilon \leq \epsilon_{\max }$ ), we have

$$
\begin{aligned}
\left\|e\left(\boldsymbol{a}_{j}\right)\right\|_{\ell^{2}} & \leq c \max _{\boldsymbol{y} \in B(\mathbf{0}, 1)}\left\|f_{h}\left(\boldsymbol{a}_{j}\right)-f_{h}\left(\boldsymbol{\varphi}_{\delta\left(\boldsymbol{a}_{j}\right)}\left(\boldsymbol{a}_{j}\right)+\delta\left(\boldsymbol{a}_{j}\right) r \boldsymbol{y}\right)\right\|_{\ell^{2}} \\
& \leq c^{\prime} \delta\left(\boldsymbol{a}_{j}\right) \max _{K^{\prime} \in \mathcal{T}_{K}}\left\|\nabla f_{h}\right\|_{\boldsymbol{L}^{\infty}\left(K^{\prime} ; \mathbb{R}^{q}\right)} \leq c^{\prime \prime} \epsilon h_{K} \max _{K^{\prime} \in \mathcal{T}_{K}}\left\|\nabla f_{h}\right\|_{\boldsymbol{L}^{\infty}\left(K^{\prime} ; \mathbb{R}^{q}\right)} .
\end{aligned}
$$

Finally using a local inverse inequality, which is legitimate since the mesh sequence is shape-regular, we infer that $\left|\sigma_{K, i}(e)\right| \leq c \epsilon\left\|\mathbb{A}_{K}\right\|_{\ell^{2}}\left\|f_{h}\right\|_{L^{\infty}\left(D_{K} ; \mathbb{R}^{q}\right)}$. Note that the purpose of the above argument is to account for the fact that $f_{h}$ is (a priori) only piecewise Lipschitz (i.e., can be discontinuous across interfaces) but $f_{h}$ is necessarily continuous at $\boldsymbol{a}_{j}$.

(3) If the degree of freedom $\sigma_{K, i}$ is an integral over an edge, face or over $K$, we use (5.12), i.e., $\left|\sigma_{K, i}(e)\right| \leq c\left\|\mathbb{A}_{K}\right\|_{\ell^{2}} \frac{1}{\left|S_{K, i}\right|} \int_{S_{K, i}}\|e\|_{\ell^{2}} \mathrm{~d} s$. We define $\mathcal{T}_{S_{K, i}}=\left\{K^{\prime} \in\right.$ $\left.\mathcal{T}_{K} \mid S_{K, i} \subset K^{\prime}\right\}$ and we introduce $S_{K, i}^{\circ}=\left\{\boldsymbol{x} \in S_{K, i} \mid \boldsymbol{\varphi}_{\delta(\boldsymbol{x})}(\boldsymbol{x})+\delta(\boldsymbol{x}) r B(\mathbf{0}, 1) \subset\right.$ $\left.\mathcal{T}_{S_{K, i}}\right\}$ and $S_{K, i}^{\partial}=S_{K, i} \backslash S_{K, i}^{\circ}$. Then using (5.14b) and setting $\boldsymbol{\psi}_{\delta}(\boldsymbol{x}, \boldsymbol{y})=\boldsymbol{\varphi}_{\delta(\boldsymbol{x})}(\boldsymbol{x})+$ $\delta(\boldsymbol{x}) r \boldsymbol{y}$, we have

$$
\begin{aligned}
\int_{S_{K, i}^{\circ}}\|e\|_{\ell^{2}} \mathrm{~d} s & \leq \int_{S_{K, i}^{\circ}} \int_{B(\mathbf{0}, 1)} \rho(\boldsymbol{y})\left\|f_{h}\left(\boldsymbol{\psi}_{\delta}(\boldsymbol{x}, \boldsymbol{y})\right)-f_{h}(\boldsymbol{x})\right\|_{\ell^{2}} \mathrm{~d} y \mathrm{~d} s \\
& \leq \int_{S_{K, i}^{\circ}} \sum_{K^{\prime} \in \mathcal{T}_{S_{K, i}}} \int_{\substack{\boldsymbol{y} \in B(\mathbf{0}, 1) \\
\boldsymbol{\psi}_{\delta}(\boldsymbol{x}, \boldsymbol{y}) \in K^{\prime}}}\left\|f_{h}\left(\boldsymbol{\psi}_{\delta}(\boldsymbol{x}, \boldsymbol{y})\right)-f_{h}(\boldsymbol{x})\right\|_{\ell^{2}} \mathrm{~d} s \mathrm{~d} y \\
& \leq c\left|S_{K, i}\right| \epsilon h_{K} \sum_{K^{\prime} \in \mathcal{T}_{S_{K, i}}}\left\|\nabla f_{h}\right\|_{\boldsymbol{L}^{\infty}\left(K^{\prime} ; \mathbb{R}^{q}\right)} \leq c\left|S_{K, i}\right| \epsilon\left\|f_{h}\right\|_{L^{\infty}\left(D_{K} ; \mathbb{R}^{q}\right)},
\end{aligned}
$$

where we used the shape-regularity of the mesh sequence (i.e., $h_{K^{\prime}} \leq c h_{K}$ ) and an inverse inequality. Note again that the above construction is meant to account for the fact that $f_{h}$ is (a priori) only piecewise Lipschitz. Moreover, if $\boldsymbol{x} \in S_{K, i}^{\partial}$, then there is $\boldsymbol{y} \in B(\mathbf{0}, 1)$ such that $\boldsymbol{z}:=\boldsymbol{\varphi}_{\delta(\boldsymbol{x})}(\boldsymbol{x})+\delta(\boldsymbol{x}) r \boldsymbol{y}$ is not in $\mathcal{T}_{S_{K, i}}$; then mesh-regularity implies that $c \operatorname{dist}\left(\boldsymbol{x}, \partial S_{K, i}\right) \leq\|\boldsymbol{z}-\boldsymbol{x}\|_{\ell^{2}}$ and that $\|\boldsymbol{z}-\boldsymbol{x}\|_{\ell^{2}} \leq c \delta(\boldsymbol{x}) \leq c^{\prime} \epsilon h_{K}$. Combining these bounds, we obtain that $\left|S_{K, i}^{\partial}\right| \leq c \epsilon h_{K}\left|\partial S_{K, i}\right| \leq c^{\prime} \epsilon\left|S_{K, i}\right|$ (with the convention that the 0 -dimensional measure of a point is 1 ). As a result, we infer that

$$
\begin{aligned}
\int_{S_{K, i}^{\partial}}\|e\|_{\ell^{2}} \mathrm{~d} s & \leq \int_{S_{K, i}^{\partial}}\left(\left\|f_{h}\right\|_{\ell^{2}}+\left\|\mathcal{K}_{\delta} f_{h}\right\|_{\ell^{2}}\right) \mathrm{d} s \\
& \leq c\left\|f_{h}\right\|_{L^{\infty}\left(D_{K} ; \mathbb{R}^{q}\right)}\left|S_{K, i}^{\partial}\right| \leq c^{\prime} \epsilon\left|S_{K, i}\right|\left\|f_{h}\right\|_{L^{\infty}\left(D_{K} ; \mathbb{R}^{q}\right)} .
\end{aligned}
$$


Combining the above two estimates yields $\left|\sigma_{K, i}(e)\right| \leq c \epsilon\left\|\mathbb{A}_{K}\right\|_{\ell^{2}}\left\|f_{h}\right\|_{L^{\infty}\left(D_{K} ; \mathbb{R}^{q}\right)}$.

(4) We have established that $\left|\sigma_{K, i}(e)\right| \leq c \epsilon\left\|\mathbb{A}_{K}\right\|_{\ell^{2}}\left\|f_{h}\right\|_{L^{\infty}\left(D_{K} ; \mathbb{R}^{q}\right)}$ for all possible degrees of freedom. Using the fact that $\left\|\mathbb{A}_{K}\right\|_{\ell^{2}}\left\|\mathbb{A}_{K}^{-1}\right\|_{\ell^{2}}$ is uniformly bounded together with an inverse inequality from $L^{\infty}\left(D_{K} ; \mathbb{R}^{q}\right)$ to $L^{p}\left(D_{K} ; \mathbb{R}^{q}\right)$, we deduce that

$$
\begin{aligned}
\left\|f_{h}-\mathcal{I}_{h} \mathcal{K}_{\delta} f_{h}\right\|_{L^{p}\left(K ; \mathbb{R}^{q}\right)} & =\left\|e_{h}\right\|_{L^{p}\left(K ; \mathbb{R}^{q}\right)} \\
& \leq c \epsilon \operatorname{det}\left(\mathbb{J}_{K}\right)^{\frac{1}{p}}\left\|\mathbb{A}_{K}^{-1}\right\|\left\|_{\ell^{2}}\right\| \mathbb{A}_{K}\left\|_{\ell^{2}}\right\| f_{h} \|_{L^{\infty}\left(D_{K} ; \mathbb{R}^{q}\right)} \\
& \leq c \epsilon\left\|f_{h}\right\|_{L^{p}\left(D_{K} ; \mathbb{R}^{q}\right)} .
\end{aligned}
$$

We infer the desired result by summing over $K \in \mathcal{T}_{h}$ and by invoking the shaperegularity of the mesh sequence.

The above lemma implies that $\left\|\left(\mathbb{I}-\mathcal{I}_{h} \mathcal{K}_{\delta}\right)_{\mid P\left(\mathcal{T}_{h}\right)}\right\|_{\mathcal{L}\left(L^{p} ; L^{p}\right)} \leq c_{\mathrm{stab}} \epsilon$ for all $\epsilon \epsilon$ $\left(0, \epsilon_{\max }\right]$. From now on we choose $\epsilon$ once and for all by setting $\epsilon=\epsilon_{\min }$ with $\epsilon_{\min }:=$ $\min \left(\epsilon_{\max },\left(2 c_{\text {stab }}\right)^{-1}\right)$. Lemma 6.1 then implies that

$$
\left\|\left(\mathbb{I}-\mathcal{I}_{h} \mathcal{K}_{\delta}\right)_{\mid P\left(\mathcal{T}_{h}\right)}\right\|_{\mathcal{L}\left(L^{p} ; L^{p}\right)} \leq \frac{1}{2}
$$

This proves that $\mathcal{I}_{h} \mathcal{K}_{\delta \mid P\left(\mathcal{T}_{h}\right)}$ is invertible for this particular choice of $\epsilon$. Let $J_{h}$ : $P\left(\mathcal{T}_{h}\right) \longrightarrow P\left(\mathcal{T}_{h}\right)$ be the inverse of $\mathcal{I}_{h} \mathcal{K}_{\delta \mid P\left(\mathcal{T}_{h}\right)}$, i.e.,

$$
J_{h} \mathcal{I}_{h} \mathcal{K}_{\delta \mid P\left(\mathcal{T}_{h}\right)}=\mathcal{I}_{h} \mathcal{K}_{\delta \mid P\left(\mathcal{T}_{h}\right)} J_{h}=\mathbb{I} .
$$

Note that the definition of $J_{h}$ implies that $\left\|J_{h}\right\|_{\mathcal{L}\left(L^{p} ; L^{p}\right)} \leq 2$.

Lemma 6.2 ( $L^{p}$-stability). Let $\epsilon=\epsilon_{\min }$. There is $c\left(\epsilon_{\min }\right)$, uniform with respect to $h$, such that the following estimate holds: $\left\|\mathcal{I}_{h} \mathcal{K}_{\delta}\right\|_{\mathcal{L}\left(L^{p} ; L^{p}\right)} \leq c\left(\epsilon_{\min }\right)$.

Proof. Let $f \in L^{p}\left(D ; \mathbb{R}^{q}\right)$ and assume $p<\infty$. Then

$$
\begin{aligned}
\left\|\mathcal{I}_{h} \mathcal{K}_{\delta} f\right\|_{L^{p}\left(D ; \mathbb{R}^{q}\right)}^{p} & =\sum_{K \in \mathcal{T}_{h}} \int_{K}\left\|\sum_{i \in \mathcal{N}} \sigma_{K, i}\left(\mathcal{K}_{\delta} f\right) \theta_{K, i}(\boldsymbol{x})\right\|_{\ell^{2}}^{p} \mathrm{~d} x \\
& \leq c \sum_{K \in \mathcal{T}_{h}} \int_{K} \sum_{i \in \mathcal{N}}\left|\sigma_{K, i}\left(\mathcal{K}_{\delta} f\right)\right|^{p}\left\|\theta_{K, i}(\boldsymbol{x})\right\|_{\ell^{2}}^{p} \mathrm{~d} x .
\end{aligned}
$$

Using (5.12), we infer that

$$
\left\|\mathcal{I}_{h} \mathcal{K}_{\delta} f\right\|_{L^{p}\left(D ; \mathbb{R}^{q}\right)}^{p} \leq c \sum_{K \in \mathcal{T}_{h}} \sum_{i \in \mathcal{N}}\left\|\mathbb{A}_{K}\right\|_{\ell^{2}}^{p}\left\|\mathcal{K}_{\delta} f\right\|_{L^{\infty}\left(K ; \mathbb{R}^{q}\right)}^{p}\left\|\mathbb{A}_{K}^{-1}\right\|_{\ell^{2}}^{p}|K|
$$

since $\left|\sigma_{K, i}\left(\mathcal{K}_{\delta} f\right)\right| \leq c\left\|\mathbb{A}_{K}\right\|_{\ell^{2}}\left\|\mathcal{K}_{\delta} f\right\|_{L^{\infty}\left(K ; \mathbb{R}^{q}\right)}$ and $\left\|\theta_{K, i}(\boldsymbol{x})\right\|_{L^{\infty}\left(K ; \mathbb{R}^{q}\right)} \leq c\left\|\mathbb{A}_{K}^{-1}\right\|_{\ell^{2}}$. We conclude by invoking Lemma 6.3 below. The argument for $p=\infty$ is similar.

LEMMA 6.3 (Local inverse inequality). Let $\epsilon=\epsilon_{\min }$. There is a uniform constant $c>0$ such that

$$
\left\|\mathcal{K}_{\delta} f\right\|_{L^{\infty}\left(K ; \mathbb{R}^{q}\right)} \leq c \epsilon_{\min }^{-d}|K|^{-\frac{1}{p}}\|f\|_{L^{p}\left(D_{K} ; \mathbb{R}^{q}\right)},
$$

for all $K \in \mathcal{T}_{h}$, all $h>0$, and all $f \in L^{p}\left(D ; \mathbb{R}^{q}\right)$.

Proof. Let $\boldsymbol{x} \in K$. Since the function $\rho$ is bounded, we infer that

$$
\left\|\mathcal{K}_{\delta} f(\boldsymbol{x})\right\|_{\ell^{2}} \leq c \int_{B(\mathbf{0}, 1)}\left\|f\left(\boldsymbol{\varphi}_{\delta(\boldsymbol{x})}(\boldsymbol{x})+\delta(\boldsymbol{x}) r \boldsymbol{y}\right)\right\|_{\ell^{2}} \mathrm{~d} y .
$$

The condition (5.14b) implies that

$$
\left\|\mathcal{K}_{\delta} f(\boldsymbol{x})\right\|_{\ell^{2}} \leq c\left\|\delta^{-1}\right\|_{L^{\infty}\left(D_{K}\right)}^{d} \int_{D_{K}}\|f(\boldsymbol{z})\|_{\ell^{2}} \mathrm{~d} z \leq c \epsilon_{\min }^{-d} h_{K}^{-d}\left|D_{K}\right|^{1-\frac{1}{p}}\|f\|_{L^{p}\left(D_{K} ; \mathbb{R}^{q}\right)},
$$

and we conclude using the shape-regularity of the mesh sequence. 
6.3. Main result. We now define the following operator

$$
\mathcal{J}_{h}=J_{h} \mathcal{I}_{h} \mathcal{K}_{\delta}
$$

and we state the main result of this section.

Theorem 6.4 (Properties of $\mathcal{J}_{h}$ ). The following properties hold:

(i) $P\left(\mathcal{T}_{h}\right)$ is point-wise invariant under $\mathcal{J}_{h}$;

(ii) There is c, uniform with respect to $h$, such that $\left\|\mathcal{J}_{h}\right\|_{\mathcal{L}\left(L^{p} ; L^{p}\right)} \leq c$ and

$$
\left\|f-\mathcal{J}_{h} f\right\|_{L^{p}\left(D ; \mathbb{R}^{q}\right)} \leq c \inf _{f_{h} \in P\left(\mathcal{T}_{h}\right)}\left\|f-f_{h}\right\|_{L^{p}\left(D ; \mathbb{R}^{q}\right)},
$$

for all $f \in L^{p}\left(D ; \mathbb{R}^{q}\right)$;

(iii) $\mathcal{J}_{h}$ commutes with the standard differential operators, i.e., the following diagrams are commutative:

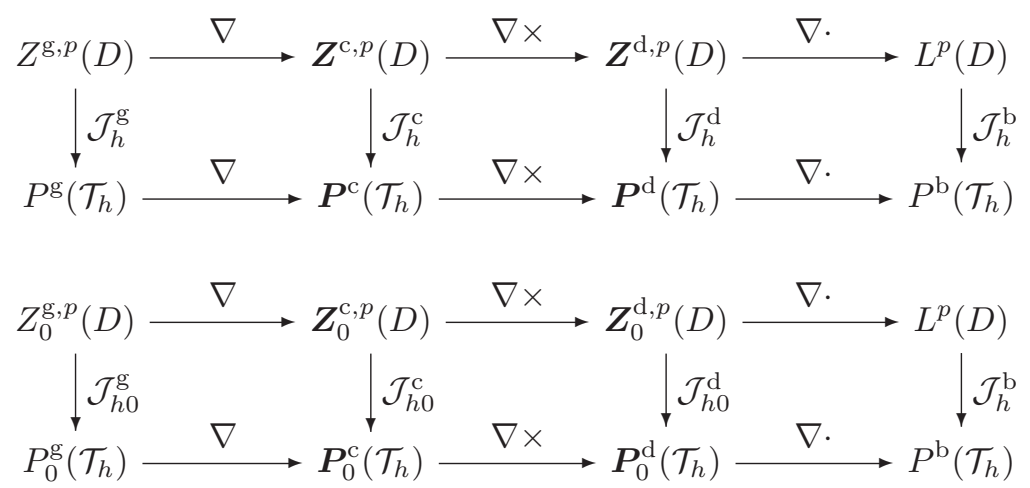

Proof. The first property is a consequence of $\mathcal{J}_{h \mid P\left(\mathcal{T}_{h}\right)}=\mathbb{I}$, since $\mathcal{J}_{h} \circ \mathcal{J}_{h}=$ $\mathcal{J}_{h \mid P\left(\mathcal{T}_{h}\right)^{\circ}} \mathcal{J}_{h}=\mathcal{J}_{h}$. The second property is proved by observing that the $L^{p}$-operatornorm of $J_{h}$ is bounded by 2 and that of $\mathcal{I}_{h} \mathcal{K}_{\delta}$ is also uniformly bounded, as established in Lemma 6.2, since $\epsilon$ is now a fixed real number. Moreover, using that $\mathcal{J}_{h} f_{h}=f_{h}$ for all $f_{h} \in P\left(\mathcal{T}_{h}\right)$, we have

$$
\begin{aligned}
& \left\|f-\mathcal{J}_{h} f\right\|_{L^{p}\left(D ; \mathbb{R}^{q}\right)}=\inf _{f_{h} \in P\left(\mathcal{T}_{h}\right)}\left\|f-f_{h}-\mathcal{J}_{h}\left(f-f_{h}\right)\right\|_{L^{p}\left(D ; \mathbb{R}^{q}\right)} \\
& \quad \leq \inf _{f_{h} \in P\left(\mathcal{T}_{h}\right)}\left(1+\left\|\mathcal{J}_{h}\right\|_{\mathcal{L}\left(L^{p} ; L^{p}\right)}\right)\left\|f-f_{h}\right\|_{L^{p}\left(D ; \mathbb{R}^{q}\right)} \leq c \inf _{f_{h} \in P\left(\mathcal{T}_{h}\right)}\left\|f-f_{h}\right\|_{L^{p}\left(D ; \mathbb{R}^{q}\right)}
\end{aligned}
$$

which establishes (ii). Let us now prove (iii). We are just going to show that the leftmost square commutes in the top diagram; the proof for the other squares is identical, and whether boundary conditions are imposed or not is immaterial in the argument. Let us first show that $J_{h}^{\mathrm{c}} \nabla \phi_{h}=\nabla\left(J_{h}^{\mathrm{g}} \phi_{h}\right)$ for all $\phi_{h} \in P^{\mathrm{g}}\left(\mathcal{T}_{h}\right)$. We observe that

$$
\nabla \phi_{h}=\nabla\left(\mathcal{I}_{h}^{\mathrm{g}} \mathcal{K}_{\delta \mid P^{\mathrm{g}}\left(\mathcal{T}_{h}\right)}^{\mathrm{g}} J_{h}^{\mathrm{g}} \phi_{h}\right)=\nabla\left(\mathcal{I}_{h}^{\mathrm{g}} \mathcal{K}_{\delta}^{\mathrm{g}} J_{h}^{\mathrm{g}} \phi_{h}\right)=\mathcal{I}_{h}^{\mathrm{c}} \nabla\left(\mathcal{K}_{\delta}^{\mathrm{g}} J_{h}^{\mathrm{g}} \phi_{h}\right)=\mathcal{I}_{h}^{\mathrm{c}} \mathcal{K}_{\delta}^{\mathrm{c}} \nabla\left(J_{h}^{\mathrm{g}} \phi_{h}\right),
$$

where we have used that $\mathbb{I}=\mathcal{I}_{h}^{\mathrm{g}} \mathcal{K}_{\delta \mid P^{\mathrm{g}}\left(\mathcal{T}_{h}\right)}^{\mathrm{g}} J_{h}^{\mathrm{g}}($ see $(6.3))$, then $\mathcal{I}_{h}^{\mathrm{g}} \mathcal{K}_{\delta \mid P^{\mathrm{g}}\left(\mathcal{T}_{h}\right)}^{\mathrm{g}} J_{h}^{\mathrm{g}}=\mathcal{I}_{h}^{\mathrm{g}} \mathcal{K}_{\delta}^{\mathrm{g}} J_{h}^{\mathrm{g}}$ (the range of $J_{h}^{\mathrm{g}}$ is in $P^{\mathrm{g}}\left(\mathcal{T}_{h}\right)$ ), followed by $\nabla \mathcal{I}_{h}^{\mathrm{g}}=\mathcal{I}_{h}^{\mathrm{c}} \nabla$ (see diagram (5.28)) and $\nabla \mathcal{K}_{\delta}^{\mathrm{g}}=\mathcal{K}_{\delta}^{\mathrm{c}} \nabla$ (see diagram $\left.(3.7)\right)$. Since $\nabla\left(J_{h}^{\mathrm{g}} \phi_{h}\right) \in \boldsymbol{P}^{\mathrm{c}}\left(\mathcal{T}_{h}\right)$ (see diagram (5.28)), the above argument together with (6.3) proves that

$$
\nabla \phi_{h}=\left(\mathcal{I}_{h}^{\mathrm{c}} \mathcal{K}_{\delta \mid \boldsymbol{P}^{\mathrm{c}}\left(\mathcal{T}_{h}\right)}^{\mathrm{c}}\right) \nabla\left(J_{h}^{\mathrm{g}} \phi_{h}\right)=\left(J_{h}^{\mathrm{c}}\right)^{-1} \nabla\left(J_{h}^{\mathrm{g}} \phi_{h}\right) .
$$


In conclusion, $J_{h}^{\mathrm{c}} \nabla \phi_{h}=\nabla\left(J_{h}^{\mathrm{g}} \phi_{h}\right)$. Now we finish the proof by using an arbitrary function $\phi \in V^{\mathrm{g}}(D)$. We have

$$
\mathcal{J}_{h}^{\mathrm{c}} \nabla \phi=J_{h}^{\mathrm{c}} \mathcal{I}_{h}^{\mathrm{c}} \mathcal{K}_{\delta}^{\mathrm{c}} \nabla \phi=J_{h}^{\mathrm{c}} \mathcal{I}_{h}^{\mathrm{c}} \nabla\left(\mathcal{K}_{\delta}^{\mathrm{g}} \phi\right)=J_{h}^{\mathrm{c}} \nabla\left(\mathcal{I}_{h}^{\mathrm{g}} \mathcal{K}_{\delta}^{\mathrm{g}} \phi\right)=\nabla\left(J_{h}^{\mathrm{g}} \mathcal{I}_{h}^{\mathrm{g}} \mathcal{K}_{\delta}^{\mathrm{g}} \phi\right)
$$

The last equality results from the fact that $J_{h}^{\mathrm{c}} \nabla \phi_{h}=\nabla\left(J_{h}^{\mathrm{g}} \phi_{h}\right)$ for all $\phi_{h} \in P^{\mathrm{g}}\left(\mathcal{T}_{h}\right)$, as established above. This proves that $\mathcal{J}_{h}^{\mathrm{c}} \nabla \phi=\nabla \mathcal{J}_{h}^{\mathrm{g}} \phi$.

Remark 6.2. (Approximation) Theorem 6.4(ii) shows that the quasi-interpolation error is bounded by the best approximation error. Estimates of best approximation errors in fractional-order Sobolev spaces have been obtained recently in Ern and Guermond [16] for general finite element spaces. As an illustration, consider a $\boldsymbol{P}^{\mathrm{c}}\left(\mathcal{T}_{h}\right)$-based finite element approximation of a field $\boldsymbol{A} \in \boldsymbol{Z}^{\mathrm{c}, p}(D)$ (typically, with $p=2)$. Suppose that the natural stability norm for this problem is that of $\boldsymbol{H}$ (curl) and that the finite element solution $\boldsymbol{A}_{h} \in \boldsymbol{P}^{\mathrm{c}}\left(\mathcal{T}_{h}\right)$ satisfies the a priori error estimate $\left\|\boldsymbol{A}-\boldsymbol{A}_{h}\right\|_{\boldsymbol{H}(\mathrm{curl})} \leq c \inf _{\boldsymbol{a}_{h} \in \boldsymbol{P}^{\mathrm{c}}\left(\mathcal{T}_{h}\right)}\left\|\boldsymbol{A}-\boldsymbol{a}_{h}\right\|_{\boldsymbol{H}(\mathrm{curl})}$. Then, taking $\boldsymbol{a}_{h}=\mathcal{J}_{h}^{\mathrm{c}} \boldsymbol{A}$ and using the commuting property leads to the bound

$$
\left\|\boldsymbol{A}-\boldsymbol{A}_{h}\right\|_{\boldsymbol{H}(\mathrm{curl})} \leq c\left(\left\|\boldsymbol{A}-\mathcal{J}_{h}^{\mathrm{c}} \boldsymbol{A}\right\|_{\boldsymbol{L}^{2}(D)}+\left\|\nabla \times \boldsymbol{A}-\mathcal{J}_{h}^{\mathrm{d}}(\nabla \times \boldsymbol{A})\right\|_{\boldsymbol{L}^{2}(D)}\right) .
$$

Assume that $\boldsymbol{A}, \nabla \times \boldsymbol{A} \in \boldsymbol{H}^{r}(D)$ for some real number $r \in(0, k+1]$ where $k$ is the degree of the finite elements composing $\boldsymbol{P}^{\mathrm{c}}\left(\mathcal{T}_{h}\right)$. Then, using Theorem 6.4(ii) together with [16, Cor.5.4] leads to $\left\|\boldsymbol{A}-\boldsymbol{A}_{h}\right\|_{\boldsymbol{H}(\text { curl })} \leq \operatorname{ch}^{r}\left(|\boldsymbol{A}|_{\boldsymbol{H}^{r}(D)}+|\nabla \times \boldsymbol{A}|_{\boldsymbol{H}^{r}(D)}\right)$. Note that no lower bound on $r$ is assumed a priori.

6.4. Discrete Poincaré inequalities. We illustrate the usefulness of the operators constructed above by proving discrete Poincaré inequalities for $\boldsymbol{H}$ (curl)-elements in dimension $d=2,3$; we expose the material for $d=3$. Assume also that $D$ is partitioned into $M$ connected, strongly Lipschitz subdomains $D_{1}, \cdots, D_{M}$. We consider two piecewise-smooth second-order tensor fields $\mathbb{E}$ and $\mu$, i.e., we assume that these fields are in

$$
\mathbb{W}^{1, \infty}\left(\cup_{i=1}^{M} D_{i}\right):=\left\{\mathbb{\sim} \in \mathbb{L}^{\infty}(D) \mid \nabla\left(\mathbb{D}_{\mid D_{i}}\right) \in\left[\mathbb{L}^{\infty}\left(D_{i}\right)\right]^{d}, i=1, \cdots, M\right\},
$$

where $\mathbb{L}^{\infty}(E):=L^{\infty}\left(E ; \mathbb{R}^{d \times d}\right)$. We additionally assume that $\mathbb{E}$ and $\mu$ are symmetric and the smallest eigenvalue of each of these two tensors is bounded away from zero from below uniformly over $D$. Consider the following Maxwell eigenvalue problems: Find $\boldsymbol{E}$ and $0 \neq \omega \in \mathbb{R}$ such that

$$
\nabla \times\left(\mu^{-1} \nabla \times \boldsymbol{E}\right)=\omega \boldsymbol{E}, \quad \nabla \cdot(\Subset \boldsymbol{E})=0, \quad \boldsymbol{E} \times \boldsymbol{n}_{\mid \partial D}=\mathbf{0},
$$

Find $\boldsymbol{B}$ and $0 \neq \omega \in \mathbb{R}$ such that

$$
\nabla \times\left(\mu^{-1} \nabla \times \boldsymbol{B}\right)=\omega \boldsymbol{B}, \quad \nabla \cdot(\mathbb{E} \boldsymbol{B})=0, \quad(\mathbb{E} \boldsymbol{B}) \cdot \boldsymbol{n}_{\mid \partial D}=0 .
$$

Upon setting $\boldsymbol{H}_{\times \boldsymbol{n}}:=\left\{\boldsymbol{z} \in \boldsymbol{H}(\mathrm{curl}) \mid \nabla \cdot(\mathbb{z})=0, \boldsymbol{z} \times \boldsymbol{n}_{\mid \partial D}=\mathbf{0}\right\}, \boldsymbol{H}_{\cdot \boldsymbol{n}}:=\{\boldsymbol{z} \in$ $\left.\boldsymbol{H}(\operatorname{curl}) \mid \nabla \cdot(\mathbb{E} \boldsymbol{z})=0,(\mathbb{E} \boldsymbol{z}) \cdot \boldsymbol{n}_{\mid \partial D}=0\right\}$, the $L^{2}$-theory of the well-posedness of this problem is based on the following embedding inequality: There are $c>0$ and $s>0$ (both depending on $D$ and $\mathbb{E}$ ) such that

$$
\begin{array}{r}
\|\boldsymbol{e}\|_{\boldsymbol{H}^{s}(D)} \leq c\|\nabla \times \boldsymbol{e}\|_{\boldsymbol{L}^{2}(D)}, \quad \forall \boldsymbol{e} \in \boldsymbol{H}_{\times \boldsymbol{n}}, \\
\|\boldsymbol{b}\|_{\boldsymbol{H}^{s}(D)} \leq c\|\nabla \times \boldsymbol{b}\|_{\boldsymbol{L}^{2}(D)}, \quad \forall \boldsymbol{b} \in \boldsymbol{H}_{\cdot \boldsymbol{n}}
\end{array}
$$


provided $\partial D$ is connected and $D$ is simply connected, respectively. The above inequalities, proved in Bonito et al. [6], generalize classical inequalities established by Costabel [14] and Birman and Solomyak [4] assuming that the tensor $\mathbb{E}$ is smooth over the entire domain.

Let us consider the finite element approximation of the above eigenvalue problem using the setting described in the previous sections. The approximation theory of this problem is non-trivial, especially when using finite elements that do not fit the De Rham Diagram. We refer to the book of Monk [26] and the review by Hiptmair [21] for an overview on the topic.

Let $\boldsymbol{P}^{\mathrm{c}}\left(\mathcal{T}_{h}\right), \boldsymbol{P}_{0}^{\mathrm{c}}\left(\mathcal{T}_{h}\right)$ be defined as above. A key step for approximating (6.9) or (6.10) consists of establishing the following discrete Poincaré inequalities: There is $c>0$, uniform with respect to $h$, such that

$$
\begin{array}{r}
\left\|\boldsymbol{e}_{h}\right\|_{\boldsymbol{L}^{2}(D)} \leq c\left\|\nabla \times \boldsymbol{e}_{h}\right\|_{\boldsymbol{L}^{2}(D)}, \quad \forall \boldsymbol{e}_{h} \in \boldsymbol{H}_{h, \times \boldsymbol{n}}, \\
\left\|\boldsymbol{b}_{h}\right\|_{\boldsymbol{L}^{2}(D)} \leq c\left\|\nabla \times \boldsymbol{b}_{h}\right\|_{\boldsymbol{L}^{2}(D)}, \quad \forall \boldsymbol{b}_{h} \in \boldsymbol{H}_{h, \cdot \boldsymbol{n}},
\end{array}
$$

where $\boldsymbol{H}_{h, \times \boldsymbol{n}}:=\left\{\boldsymbol{v}_{h} \in \boldsymbol{P}_{0}^{\mathrm{c}}\left(\mathcal{T}_{h}\right) \mid \int_{D}\left(\mathbb{E} \boldsymbol{v}_{h}\right) \cdot \nabla q_{h} \mathrm{~d} x=0, \forall q_{h} \in P_{0}^{\mathrm{g}}\left(\mathcal{T}_{h}\right)\right\}$ and $\boldsymbol{H}_{h, \cdot \boldsymbol{n}}:=$ $\left\{\boldsymbol{v}_{h} \in \boldsymbol{P}^{\mathrm{c}}\left(\mathcal{T}_{h}\right) \mid \int_{D}\left(\mathbb{E} \boldsymbol{v}_{h}\right) \cdot \nabla q_{h} \mathrm{~d} x=0, \forall q_{h} \in P^{\mathrm{g}}\left(\mathcal{T}_{h}\right)\right\}$. There are many ways of proving (6.12a)-(6.12b) when $\mathbb{E}$ is smooth, since in this case it can be proved that the Sobolev index $s$ in (6.11a) is larger than $\frac{1}{2}$. The first route described in [21, §4.2] consists of invoking subtle regularity estimates from Amrouche et al. [1, Lemma 4.7]. The second one, which avoids invoking regularity estimates, is based on the so-called discrete compactness argument of Kikuchi [23] and further developed by Monk and Demkowicz [27] and Caorsi et al. [9]. The proof is not constructive and is based on an argument by contradiction.

We now show that using the approximation operators described in the previous sections gives a direct answer to the above question without requiring any particular condition on the Sobolev index $s$ in (6.11a); see also Arnold et al. [3, Thm 3.6].

Theorem 6.5 (Discrete Poincaré). Assume that $\partial D$ is connected (resp., $D$ is simply connected). Then there is a uniform constant $c>0$ such that (6.12a) holds (resp., (6.12b) holds).

Proof. We only do the proof for (6.12a), the proof for $(6.12 \mathrm{~b})$ is similar. Let $\boldsymbol{v}_{h} \in \boldsymbol{H}_{h, \times \boldsymbol{n}}$ be a nonzero discrete field. Let $\phi\left(\boldsymbol{v}_{h}\right) \in H_{0}^{1}(D)$ be the solution to the following Poisson problem:

$$
\nabla \cdot\left(\mathbb{E} \nabla \phi\left(\boldsymbol{v}_{h}\right)\right)=\nabla \cdot\left(\mathbb{E} \boldsymbol{v}_{h}\right), \quad \phi\left(\boldsymbol{v}_{h}\right)_{\mid \partial D}=0 .
$$

Note that this problem is well-posed owing to the assumed regularity and structure of $\mathbb{E}$. Let us define $\boldsymbol{v}\left(\boldsymbol{v}_{h}\right):=\boldsymbol{v}_{h}-\nabla \phi\left(\boldsymbol{v}_{h}\right)$. This definition implies that

$$
\nabla \cdot\left(\mathbb{E} \boldsymbol{v}\left(\boldsymbol{v}_{h}\right)\right)=0, \quad \nabla \times\left(\boldsymbol{v}\left(\boldsymbol{v}_{h}\right)\right)=\nabla \times \boldsymbol{v}_{h}, \quad \boldsymbol{v}\left(\boldsymbol{v}_{h}\right) \times \boldsymbol{n}_{\mid \partial D}=\mathbf{0},
$$

so that $\boldsymbol{v}\left(\boldsymbol{v}_{h}\right) \in \boldsymbol{H}_{\times \boldsymbol{n}}$. We now bound $\left\|\boldsymbol{v}_{h}\right\|_{\boldsymbol{L}^{2}(D)}$ as follows:

$$
\begin{aligned}
c\left\|\boldsymbol{v}_{h}\right\|_{L^{2}(D)}^{2} & \leq \int_{D}\left(\mathbb{E} \boldsymbol{v}_{h}\right) \cdot \boldsymbol{v}_{h} \mathrm{~d} x=\int_{D}\left(\mathbb{E} \boldsymbol{v}_{h}\right) \cdot\left(\boldsymbol{v}_{h}-\mathcal{J}_{h 0}^{\mathrm{c}} \boldsymbol{v}\left(\boldsymbol{v}_{h}\right)+\mathcal{J}_{h 0}^{\mathrm{c}} \boldsymbol{v}\left(\boldsymbol{v}_{h}\right)\right) \mathrm{d} x \\
& =\int_{D}\left(\mathbb{E} \boldsymbol{v}_{h}\right) \cdot \mathcal{J}_{h 0}^{\mathrm{c}}\left(\boldsymbol{v}_{h}-\boldsymbol{v}\left(\boldsymbol{v}_{h}\right)\right) \mathrm{d} x+\int_{D}\left(\mathbb{E} \boldsymbol{v}_{h}\right) \cdot \mathcal{J}_{h 0}^{\mathrm{c}} \boldsymbol{v}\left(\boldsymbol{v}_{h}\right) \mathrm{d} x \\
& =\int_{D}\left(\mathbb{E} \boldsymbol{v}_{h}\right) \cdot \mathcal{J}_{h 0}^{\mathrm{c}} \nabla\left(\phi\left(\boldsymbol{v}_{h}\right)\right) \mathrm{d} x+\int_{D}\left(\mathbb{E} \boldsymbol{v}_{h}\right) \cdot \mathcal{J}_{h 0}^{\mathrm{c}} \boldsymbol{v}\left(\boldsymbol{v}_{h}\right) \mathrm{d} x .
\end{aligned}
$$


Note here that we used that $\mathcal{J}_{h 0}^{\mathrm{c}} \boldsymbol{v}_{h}=\boldsymbol{v}_{h}$. Then using the commuting property $\mathcal{J}_{h 0}^{\mathrm{c}} \nabla\left(\phi\left(\boldsymbol{v}_{h}\right)\right)=\nabla\left(\mathcal{J}_{h, 0}^{\mathrm{g}} \phi\left(\boldsymbol{v}_{h}\right)\right)$ and since $\mathcal{J}_{h, 0}^{\mathrm{g}}$ maps onto $P_{0}^{\mathrm{g}}\left(\mathcal{T}_{h}\right)$, we infer that

$$
\begin{aligned}
& c\left\|\boldsymbol{v}_{h}\right\|_{\boldsymbol{L}^{2}(D)}^{2} \leq \int_{D}\left(\mathbb{(} \boldsymbol{v}_{h}\right) \cdot \nabla\left(\mathcal{J}_{h, 0}^{\mathrm{g}} \phi\left(\boldsymbol{v}_{h}\right)\right) \mathrm{d} x+\int_{D}\left(\Subset \boldsymbol{v}_{h}\right) \cdot \mathcal{J}_{h 0}^{\mathrm{c}} \boldsymbol{v}\left(\boldsymbol{v}_{h}\right) \mathrm{d} x \\
& =\int_{D}\left({ }^{\oplus} \boldsymbol{v}_{h}\right) \cdot \mathcal{J}_{h 0}^{\mathrm{c}} \boldsymbol{v}\left(\boldsymbol{v}_{h}\right) \mathrm{d} x \leq c^{\prime}\left\|\boldsymbol{v}_{h}\right\|_{L^{2}(D)}\left\|\mathcal{J}_{h 0}^{\mathrm{c}} \boldsymbol{v}\left(\boldsymbol{v}_{h}\right)\right\|_{\boldsymbol{L}^{2}(D)} .
\end{aligned}
$$

The uniform boundedness of $\mathcal{J}_{h 0}^{\mathrm{c}}$ on $\boldsymbol{L}^{2}(D)$ and (6.11a) with $s=0$ imply

$$
\left\|\boldsymbol{v}_{h}\right\|_{\boldsymbol{L}^{2}(D)} \leq c\left\|\mathcal{J}_{h 0}^{\mathrm{c}} \boldsymbol{v}\left(\boldsymbol{v}_{h}\right)\right\|_{\boldsymbol{L}^{2}(D)} \leq c^{\prime}\left\|\boldsymbol{v}\left(\boldsymbol{v}_{h}\right)\right\|_{\boldsymbol{L}^{2}(D)} \leq c^{\prime \prime}\left\|\nabla \times \boldsymbol{v}_{h}\right\|_{\boldsymbol{L}^{2}(D)} .
$$

This concludes the proof.

Acknowledgments. The authors acknowledge fruitful discussions with S. H. Christiansen and A. Demlow.

\section{References.}

[1] C. Amrouche, C. Bernardi, M. Dauge, and V. Girault. Vector potentials in three-dimensional non-smooth domains. Math. Methods Appl. Sci., 21(9):823864, 1998.

[2] D. N. Arnold, R. S. Falk, and R. Winther. Finite element exterior calculus, homological techniques, and applications. Acta Numer., 15:1-155, 2006.

[3] D. N. Arnold, R. S. Falk, and R. Winther. Finite element exterior calculus: from Hodge theory to numerical stability. Bull. Amer. Math. Soc. (N.S.), 47(2): 281-354, 2010.

[4] M. S. Birman and M. Z. Solomyak. $L_{2}$-theory of the Maxwell operator in arbitrary domains. Uspekhi Mat. Nauk, 42(6(258)):61-76, 247, 1987.

[5] A. Blouza and H. Le Dret. An up-to-the-boundary version of Friedrichs's lemma and applications to the linear Koiter shell model. SIAM J. Math. Anal., 33(4): 877-895 (electronic), 2001.

[6] A. Bonito, J.-L. Guermond, and F. Luddens. Regularity of the Maxwell equations in heterogeneous media and Lipschitz domains. J. Math. Anal. Appl., 408(2): 498-512, 2013.

[7] A. Bonito, J.-L. Guermond, and F. Luddens. An interior penalty method with $C^{0}$ finite elements for the approximation of the Maxwell equations in heterogeneous media: Convergence analysis with minimal regularity. M2AN Math. Model. Numer. Anal., 2015. In press, arXiv:1402.4454.

[8] H. Brezis. Functional analysis, Sobolev spaces and partial differential equations. Universitext. Springer, New York, 2011.

[9] S. Caorsi, P. Fernandes, and M. Raffetto. On the convergence of Galerkin finite element approximations of electromagnetic eigenproblems. SIAM J. Numer. Anal., 38(2):580-607 (electronic), 2000.

[10] S. H. Christiansen. Stability of Hodge decompositions in finite element spaces of differential forms in arbitrary dimension. Numer. Math., 107(1):87-106, 2007.

[11] S. H. Christiansen. Finite element systems of differential forms. Technical Report http://arxiv.org/abs/1006.4779v3, arXiv, 2015.

[12] S. H. Christiansen and R. Winther. Smoothed projections in finite element exterior calculus. Math. Comp., 77(262):813-829, 2008.

[13] P. G. Ciarlet. The finite element method for elliptic problems, volume 40 of Classics in Applied Mathematics. Society for Industrial and Applied Mathematics 
(SIAM), Philadelphia, PA, 2002. Reprint of the 1978 original [North-Holland, Amsterdam; MR0520174 (58 \#25001)].

[14] M. Costabel. A remark on the regularity of solutions of Maxwell's equations on Lipschitz domains. Math. Methods Appl. Sci., 12(4):365-368, 1990.

[15] A. Ern and J.-L. Guermond. Theory and practice of finite elements, volume 159 of Applied Mathematical Sciences. Springer-Verlag, New York, 2004.

[16] A. Ern and J.-L. Guermond. Finite element quasi-interpolation and best approximation. Technical Report http://arxiv.org/abs/1505.06931, arXiv, 2015.

[17] R. S. Falk and R. Winther. Local bounded cochain projections. Math. Comp., 83(290):2631-2656, 2014.

[18] K. O. Friedrichs. The identity of weak and strong extensions of differential operators. Trans. Amer. Math. Soc., 55:132-151, 1944.

[19] V. Girault and L. R. Scott. Analysis of a two-dimensional grade-two fluid model with a tangential boundary condition. J. Math. Pures Appl. (9), 78(10):981-1011, 1999.

[20] P. Grisvard. Elliptic problems in nonsmooth domains, volume 24 of Monographs and Studies in Mathematics. Pitman (Advanced Publishing Program), Boston, MA, 1985.

[21] R. Hiptmair. Finite elements in computational electromagnetism. Acta Numer., 11:237-339, 2002.

[22] S. Hofmann, M. Mitrea, and M. Taylor. Geometric and transformational properties of Lipschitz domains, Semmes-Kenig-Toro domains, and other classes of finite perimeter domains. J. Geom. Anal., 17(4):593-647, 2007.

[23] F. Kikuchi. On a discrete compactness property for the Nédélec finite elements. J. Fac. Sci. Univ. Tokyo Sect. IA Math., 36(3):479-490, 1989.

[24] J. Leray. Sur le mouvement d'un liquide visqueux emplissant l'espace. Acta Math., 63(1):193-248, 1934.

[25] W. McLean. Strongly elliptic systems and boundary integral equations. Cambridge University Press, Cambridge, 2000.

[26] P. Monk. Finite element methods for Maxwell's equations. Numerical Mathematics and Scientific Computation. Oxford University Press, New York, 2003.

[27] P. Monk and L. Demkowicz. Discrete compactness and the approximation of Maxwell's equations in $\mathbb{R}^{3}$. Math. Comp., 70(234):507-523, 2001.

[28] J. Schöberl. Commuting quasi-interpolation operators for mixed finite elements. Technical Report ISC-01-10-MATH, Texas A\&M University, 2001. URL www.isc. tamu.edu/publications-reports/tr/0110.pdf.

[29] J. Schöberl. A multilevel decomposition result in $H$ (curl). In P. Wesseling, C. W. Oosterlee, and P. Hemker, editors, Multigrid, Multilevel and Multiscale Methods, EMG 2005, 2005.

[30] J. Schöberl. A posteriori error estimates for Maxwell equations. Math. Comp., 77(262):633-649, 2008.

[31] S. Soboleff. Sur un théorème d'analyse fonctionnelle. Rec. Math. [Mat. Sbornik] N.S., 4(46):471-497, 1938. 